\title{
Reasoning about Topological and Cardinal Direction Relations Between 2-Dimensional Spatial Objects
}

\author{
Anthony G. Cohn \\ School of Computing, University of Leeds, UK \\ Faculty of Engineering and Information Technology, \\ University of Technology Sydney, Australia

\section{Sanjiang Li} \\ AMSS-UTS Joint Research Lab, \\ Centre for Quantum Computation \& Intelligent Systems, \\ University of Technology Sydney, Australia \\ College of Computer Science, Shaanxi Normal University, China \\ Weiming Liu \\ Baidu (China) Co., Ltd., Shanghai, China \\ Jochen Renz \\ Research School of Computer Science, \\ The Australian National University, Australia
}

A.G.CoHn@LEEDS.AC.UK

SANJIANG.LI@UTS.EDU.AU

LIUWEIMING@BAIDU.COM

JOCHEN.RENZ@ANU.EDU.AU

\begin{abstract}
Increasing the expressiveness of qualitative spatial calculi is an essential step towards meeting the requirements of applications. This can be achieved by combining existing calculi in a way that we can express spatial information using relations from multiple calculi. The great challenge is to develop reasoning algorithms that are correct and complete when reasoning over the combined information. Previous work has mainly studied cases where the interaction between the combined calculi was small, or where one of the two calculi was very simple. In this paper we tackle the important combination of topological and directional information for extended spatial objects. We combine some of the best known calculi in qualitative spatial reasoning, the RCC8 algebra for representing topological information, and the Rectangle Algebra (RA) and the Cardinal Direction Calculus (CDC) for directional information. We consider two different interpretations of the RCC8 algebra, one uses a weak connectedness relation, the other uses a strong connectedness relation. In both interpretations, we show that reasoning with topological and directional information is decidable and remains in NP. Our computational complexity results unveil the significant differences between RA and CDC, and that between weak and strong RCC8 models. Take the combination of basic RCC8 and basic CDC constraints as an example: we show that the consistency problem is in P only when we use the strong RCC8 algebra and explicitly know the corresponding basic RA constraints.
\end{abstract}

\section{Introduction}

Qualitative Spatial Reasoning (QSR) is a multi-disciplinary research field that aims at establishing expressive representation formalisms of qualitative spatial knowledge and providing effective reasoning mechanisms. Originating from Allen's work (1983) on temporal interval relations, QSR has been widely acknowledged as the AI approach to spatial knowledge representation and reasoning, with applications ranging from natural language understanding (Davis, 2013), robot navigation (Shi, Jian, \& Krieg-Brückner, 2010; Falomir, 2012), geographic information systems (GISs) (Egenhofer 
\& Mark, 1995), sea navigation (Wolter et al., 2008), to high level interpretation of video data (Sridhar, Cohn, \& Hogg, 2011; Cohn, Renz, \& Sridhar, 2012). We refer the reader to the work of Cohn and Renz (2008), and Wolter and Wallgrün (2012) for more information.

The qualitative approach usually represents spatial information by introducing a relation model on the domain of spatial entities, which could be points, line segments, rectangles, or arbitrary regions. In the literature, such a relation model is often called a qualitative calculus (Ligozat \& Renz, 2004), which contains a finite set of jointly exhaustive and pairwise disjoint (JEPD) relations defined on the domain. In the past three decades, dozens of spatial relation models have been proposed in the literature (Cohn \& Renz, 2008; Chen, Cohn, Liu, Wang, Ouyang, \& Yu, 2013). Many of these qualitative calculi approximate spatial entities by points. While this is convenient when representing spatial direction, distance and positions (providing the extent of the objects is small compared to their distance apart), it is inappropriate as far as the shapes and/or topology of the spatial objects are concerned. In this paper, we represent spatial entities as 2-dimensional bounded regions in the real plane, which may have holes or multiple connected components.

In the literature, most spatial calculi focus on one single aspect of space, e.g. topology, direction, distance, position, or shape. Topological relations are those relations that are invariant under homeomorphisms such as scale, rotation, and translation. It is widely acknowledged that topological relations are of crucial importance. One influential formalism for topological relations is the region connection calculus (RCC) (Randell, Cui, \& Cohn, 1992). Based on one primitive binary connectedness relation, a set of eight JEPD topological relations can be defined in the RCC. This calculus is known as the RCC8 algebra. According to different interpretations of connectedness, this calculus may have different variants. In this paper, we say two (closed) regions are weakly connected if they share at least a common point, and say they are strongly connected if their intersection is at least one-dimensional. Accordingly, we address the two resulting RCC8 algebras as the weak and the strong RCC8 algebras respectively. For convenience, we denote the weak RCC8 algebra as $\mathrm{RCC} 8$, and the strong one as RCC $8^{\prime}$.

The importance of the distinction between strong and weak RCC 8 becomes clear when analysing the different ways of defining the neighbourhood of pixels commonly used in Computer Vision. 4connectedness refers to the pixels that are horizontally and vertically connected to a pixel, while 8 -connectedness includes the diagonally neighbouring pixels as well. This distinction corresponds nicely to the distinction between strong and weak RCC8 as 8-connectedness considers connections at a point, while 4-connectedness only considers connections along a line (which is onedimensional). Therefore, we can use strong or weak RCC8 in a similar way we use 4- or 8connectedness, depending on the requirements of the application at hand.

The RCC 8 algebra only represents topological information between spatial objects. In many practical applications, however, other kinds of relations are often used together with topological relations. For example, when recommending a restaurant you dined at before it is common to give descriptions such as "the restaurant is in the city centre, west of the central station, and nearby there is a McDonald's."

Among all these aspects of spatial information other than topology, directional relations are perhaps the most important. There are two well-known formalisms that can cope with directional relations between extended spatial objects. One is the Rectangle Algebra (RA) (Balbiani, Condotta, \& Fariñas del Cerro, 1999), the other is the Cardinal Direction Calculus (CDC) (Goyal \& Egenhofer, 2001; Skiadopoulos \& Koubarakis, 2005). When representing the direction of a primary object to a reference object, RA approximates both the reference object and the primary object by their 
minimum bounding rectangles (MBRs), and relates the two objects by the interval relations between the projected intervals. On the other hand, CDC only approximates the reference object by its MBR, while leaving the primary object unchanged. The CDC has 511 basic relations, and RA has 169 basic relations. Most (487 out of 511) basic CDC relations intersect with one and only one basic $\mathrm{RA}$ relation and, hence, are contained in a unique basic RA relation. Therefore, CDC is in a sense more expressive than RA.

A central reasoning problem in QSR is the consistency problem. An instance of the consistency problem is a set $\Gamma$ of constraints like $(x \alpha y)$, where $x, y$ are spatial variables, and $\alpha$ is a qualitative relation from a qualitative calculus. We say $\Gamma$ is consistent or satisfiable if there exists an instantiation of the spatial variables such that all constraints in $\Gamma$ are satisfied. Without loss of generality, we assume that there is a unique constraint between any two variables. Note that if $x$ and $y$ are not related, we can add $(x \star y)$ in $\Gamma$ without changing its consistency, where $\star$ is the universal relation in the calculus. Unlike classical CSPs, the domain of a spatial variable is usually infinite, and it may be undecidable to determine the consistency of binary CSPs with infinite domains (Hirsch, 1999). In the past three decades, QSR has made significant progress in solving the consistency problems for a variety of qualitative calculi (Renz \& Nebel, 1999; Renz, 1999; Balbiani et al., 1999; Zhang, Liu, Li, \& Ying, 2008; Skiadopoulos \& Koubarakis, 2005; Liu, Zhang, Li, \& Ying, 2010; Liu \& Li, 2011).

In order to bring spatial reasoning theory closer to practical applications, it is necessary to combine multiple aspects of spatial information. A growing number of works have been devoted to combining topological RCC relations with other aspects of spatial information, e.g. qualitative size (Gerevini \& Renz, 2002), cardinal directions (Sistla \& Yu, 2000; Li, 2006a, 2007; Liu, Li, \& Renz, 2009; Li \& Cohn, 2012), connectivity (Kontchakov, Nenov, Pratt-Hartmann, \& Zakharyaschev, 2011), convexity (Davis, Gotts, \& Cohn, 1999; Schockaert \& Li, 2012), betweenness (Schockaert \& Li, 2013), and gravity (Ge \& Renz, 2013). Recently, Wölfl and Westphal (2009) also empirically compared two approaches to the combination of binary qualitative constraint calculi in general. There are also interesting works on combining spatial and temporal formalisms (Gerevini \& Nebel, 2002; Gabelaia, Kontchakov, Kurucz, Wolter, \& Zakharyaschev, 2005). Moreover, in other subareas of formalisms of constraint research, combination of formalisms has been discussed for a long time and there are some very strong results, see e.g. the work by Bodirsky and Kára (2010), and Jonsson and Krokhin (2004).

The current paper considers the full combination of RCC8 and $\mathrm{RCC} 8^{\prime}$ with the two directional relation models RA and CDC. We identify the joint satisfaction problem (JSP) as the main reasoning task. Given a network of topological (RCC8 or RCC8') constraints $\Theta$ and a network of directional (RA or CDC) constraints $\Delta$, assuming that $\Theta$ and $\Delta$ involve the same set of variables, the JSP is to decide when the joint network $\Theta \uplus \Delta$ is satisfiable. Note that we use $\uplus$, instead of $\cup$, to indicate that $\Theta$ and $\Delta$ are over the same variables.

Since topological and directional information is not independent, it is possible that the joint network $\Theta \uplus \Delta$ is unsatisfiable while both $\Theta$ and $\Delta$ are satisfiable. Solving the joint satisfaction problem is in general harder than solving $\Theta$ and $\Delta$ independently. In this paper, we interpret directional relations in terms of RA and CDC, and interpret topological relations in terms of the weak and the strong RCC8 algebras. When only basic constraints are involved, we show that the JSP for basic (weak or strong) RCC8 and basic RA networks can be solved in polynomial time, but the JSP for basic (weak or strong) RCC8 and basic CDC networks is NP-complete. Furthermore, we show that, when the three calculi (viz. RCC8, RA, and CDC) are combined together, the JSP for 
basic RCC8' networks and basic RA and CDC networks is tractable. Since non-basic constraints can always be backtracked to basic constraints, these results show that the JSP over (weak or strong) RCC8 and RA or CDC is in NP.

This paper is a significant extension of the conference paper (Liu et al., 2009), where the combination of basic weak RCC8 and RA or CDC constraints was considered. This paper also considers the combination of $\mathrm{RCC}^{\prime}$ and $\mathrm{RA}$ and/or CDC constraints. In addition, we extend our tractable results to two maximal tractable subsets of RCC8 and one large tractable subset of RA. This paper is also closely related to the work of $\mathrm{Li} \mathrm{(2007),} \mathrm{and} \mathrm{Li}$ and Cohn (2012), where the combination of the weak RCC8 algebra and two subalgebras (viz. DIR9 and DIR49) of RA is considered.

\subsection{An Application Scenario}

As an example for demonstrating the usefulness of our results, we use the Angry Birds domain. Similar representation and reasoning tasks can be applied whenever we use computer vision to detect objects in image or video. Angry Birds is a popular computer game that has gained increasing attention within the AI community, see e.g. the work of Zhang and Renz (2014). The Angry Birds AI competition is an AI challenge problem, where the goal is to build an intelligent agent that can play Angry Birds better than the best human players (see http://aibirds.org).

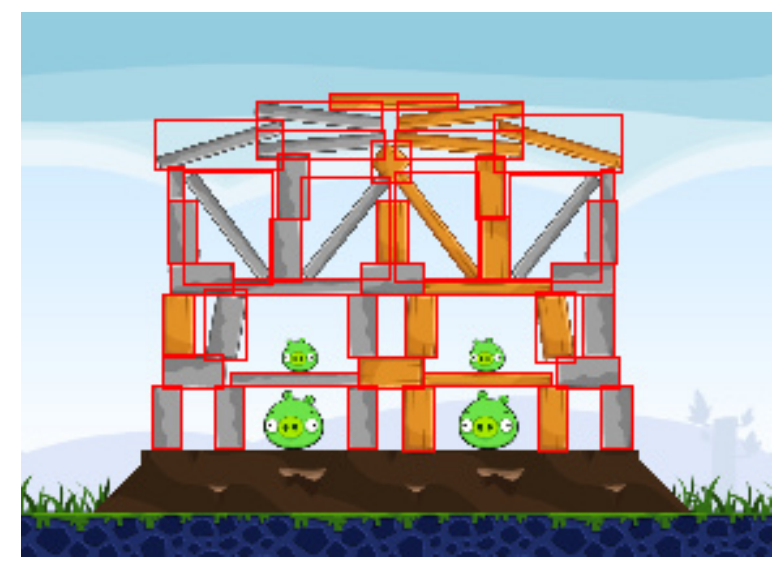

Figure 1: A screenshot of the Angry Birds game.

The Angry Birds domain includes a number of building blocks of different materials, sizes and shapes, and even with holes. The building blocks form complicated spatial structures that protect pigs from the attacking birds (see Figure 1). AI agents have to be able to play the game like humans do, that is they only get visual information about the game in the form of screenshots. The competition organisers provide a basic computer vision software that detects the minimum bounding boxes of all objects in a screenshot as well as the object category. So what is given is a set of rectangles that form the minimum bounding boxes of the actual objects (see Figure 1). While each object is a solid physical object that cannot overlap another object (only RCC8 relations DC and EC are possible between objects), their bounding boxes can be related in any relation of the Rectangle Algebra and any relation in CDC. Instead of considering only spatial relations between single objects, we can also take into account sets of objects, for example, the set of all objects that are directly or indirectly supported by a particular other object, or the set of all objects that provide cover for a particular pig, or the set of all wooden blocks. 


\begin{tabular}{c|c} 
Notations & Meanings \\
\hline$\alpha, \beta, \gamma, \delta, \theta, \rho$ & relations, usually basic relations (page 498) \\
$D, R, S, T$ & relations, usually non-basic relations (page 498) \\
$\alpha^{\sim}$ & the converse relation of $\alpha$ (page 498) \\
$\alpha \circ_{w} \beta$ & the weak composition of $\alpha$ and $\beta$ (page 498) \\
$x, y, z, v_{i}, v_{j}$ & spatial variable or interval variable (page 498) \\
$\Theta, \Gamma, \Delta$ & network of constraints (page 498) \\
$a, b, c, m$ & bounded regions (page 500) \\
$\widehat{\mathcal{H}}_{8}, \mathcal{Q}_{8}, \mathcal{C}_{8}$ & points (page 501) \\
$P, Q$ & the three maximal tractable subclasses of RCC8 (page 500) \\
$\mathcal{H}$ & the unique maximal tractable subclass of IA (page 502) \\
$I_{x}(a), I_{y}(a)$ & the $x$ - and $y$ - projective intervals of region $a$ (page 503) \\
$\mathcal{M}(a)$ & the minimal bounding rectangle (MBR) of region $a$ (page 503) \\
$\alpha \otimes \beta$ & the RA relation induced by two IA relations $\alpha, \beta$ (page 503) \\
$\mathfrak{m}=\left(m_{i}\right)_{i=1}^{n}$ & an $n$-tuple of regions $m_{i}$ that form a solution to some network (page 504) \\
$(\delta, \gamma)$ & a consistent pair of basic CDC relations (page 505) \\
$\iota^{x}(\delta, \gamma), \iota^{y}(\delta, \gamma)$ & the $x$ - and $y$ - projective interval relations of $(\delta, \gamma)$ (page 505) \\
$\iota(\delta, \gamma)$ & the RA relation $\iota^{x}(\delta, \gamma) \otimes \iota^{y}(\delta, \gamma)$ induced by $(\delta, \gamma)$ (page 506) \\
$\Theta \uplus \Gamma$ & the combination of two networks over the same set of variables (page 507) \\
$\mathbf{J S P}(\mathcal{S}, \mathcal{T})$ & the joint satisfaction problem over subclasses $\mathcal{S}$ and $\mathcal{T}$ (page 507) \\
$R A(T)$ & the RA relation induced by an RCC8 relation $T$ (page 509) \\
$R C C 8(D)$ & the RCC8 relation induced by an RA relation $D$ (page 509) \\
$\operatorname{CCP}\left(v_{i}, v_{j}\right)$ & two variables $v_{i}, v_{j}$ have the common conflict point relation (page 510)
\end{tabular}

\section{Table 1: Notations.}

These sets of building blocks form spatial regions in the general sense as used by RCC 8 and BRCC8 (Wolter \& Zakharyaschev, 2000), which also include regions with multiple disconnected pieces or regions with holes. In particular, it means that any RCC8 relation is possible between two sets of objects, not just DC or EC.

Given spatial configurations in the Angry Birds domain, we can now use RCC8 relations as well as RA and CDC relations to represent spatial information about (sets of) objects and their minimum bounding boxes that is extracted from the screenshots. The results of this paper allow us to accurately reason about the combined information represented using RCC8, RA, and CDC. Important reasoning tasks that can benefit from our results include, for example, inferring how a configuration changes after it is hit by a bird or inferring whether a given representation is consistent or whether it is stable under gravity (Zhang \& Renz, 2014). An algorithm for predicting the configuration of the blocks after a shot might work by envisaging individual possible block positions but these might be mutually or globally inconsistent. An algorithm for reasoning about the consistency of such predictions is therefore desirable.

The remainder of this paper proceeds as follows. Section 2 introduces basic notions, important examples, and essential results of qualitative calculi. Section 3 then describes the joint satisfaction problem and considers the simple example of the combination of RA and CDC constraints. Sections 4 and 5 consider the computational complexity of the combination of weak and, respectively, strong 
RCC8 with RA. Section 6 discusses the computational complexity of the combination of weak and strong RCC8 with CDC. We conclude the paper in Section 7 and give proofs of major computational complexity results in the appendices. For the convenience of the reader, Table 1 summarises notations used in this paper.

\section{Qualitative Calculi}

The establishment of a proper qualitative calculus is the key to the success of the qualitative approach to temporal and spatial reasoning. This section introduces basic notions of qualitative calculi and recalls the RCC8 algebra, the Rectangle Algebra, and the Cardinal Direction Calculus. In addition, we will also summarise some essential results that will be used in the main part of the paper.

\subsection{Basic Notions}

Let $\mathbb{U}$ be the domain of temporal or spatial entities, and $\operatorname{Re}(\mathbb{U})$ be the set of binary relations on $\mathbb{U}$. With the usual relational operations of intersection, union, and complement, $\operatorname{Re}(\mathbb{U})$ is a Boolean algebra. A finite set $\mathcal{B}$ of nonempty binary relations on $\mathbb{U}$ is jointly exhaustive and pairwise disjoint (JEPD for short) if any two entities in $\mathbb{U}$ are related by one and only one relation in $\mathcal{B}$. Write $\langle\mathcal{B}\rangle$ for the subalgebra of $\operatorname{Rel}(\mathbb{U})$ generated by $\mathcal{B}$. Clearly, relations in $\mathcal{B}$ are atoms in the algebra $\langle\mathcal{B}\rangle$. We call $\langle\mathcal{B}\rangle$ a qualitative calculus on $\mathbb{U}$, and call relations in $\mathcal{B}$ basic relations of the calculus.

Notation. Note that each relation in $\langle\mathcal{B}\rangle$ is the union of a set of basic relations. In this paper, we write $R=\left\{\alpha_{1}, \alpha_{2}, \ldots, \alpha_{k}\right\}$ if $R$ is the union of basic relations $\alpha_{1}, \alpha_{2}, \ldots, \alpha_{k}$. For convenience, we regard each basic relation $\alpha$ as the singleton $\{\alpha\}$.

For two relations $R, S$ in a qualitative calculus $\mathbf{M}=\langle\mathcal{B}\rangle$, we write $R^{\sim}$ for the converse of $R$, which is defined as

$$
R^{\sim}=\{(x, y) \in \mathbb{U} \times \mathbb{U}:(y, x) \in R\}
$$

and write $R \circ_{w} S$ for the smallest relation in $\mathbf{M}$ which contains $R \circ S$, the usual composition of $R$ and $S$, which is defined as

$$
R \circ S=\{(x, y) \in \mathbb{U}:(\exists z \in \mathbb{U})(x, z) \in R \wedge(z, y) \in S\}
$$

We call $R \circ_{w} S$ the weak composition of $R$ and $S$ (Düntsch, Wang, \& McCloskey, 2001).

A constraint over $\langle\mathcal{B}\rangle$ has the form $(x R y)$, where $R$ is a relation in $\langle\mathcal{B}\rangle$. We call $(x R y)$ a basic constraint if $R$ is a basic relation in $\mathcal{B}$. An important reasoning problem in a qualitative calculus is to determine the satisfiability or consistency of a network $\Gamma=\left\{v_{i} R_{i j} v_{j}\right\}_{i, j=1}^{n}$ of constraints over $\langle\mathcal{B}\rangle$, where $\Gamma$ is satisfiable (or consistent) if there is an instantiation $\left(a_{i}\right)_{i=1}^{n}$ in $\mathbb{U}$ such that $\left(a_{i}, a_{j}\right) \in R_{i j}$ holds for all $1 \leq i, j \leq n$.

Given two constraint networks $\Gamma=\left\{v_{i} R_{i j} v_{j}\right\}_{i, j=1}^{n}$ and $\Theta=\left\{v_{i} T_{i j} v_{j}\right\}_{i, j=1}^{n}$, we say $\Theta$ refines $\Gamma$ if $T_{i j}$ is a subset of $R_{i j}$ for any $1 \leq i, j \leq n$. A consistent scenario of $\Gamma$ is a consistent basic network that refines $\Gamma$. It is clear that $\Gamma$ is consistent iff it has a consistent scenario. On the other hand, given an $n$-tuple of entities $\left(a_{i}\right)_{i=1}^{n}$ in $\mathbb{U}$, write $\delta_{i j}$ for the basic relation in a fixed qualitative calculus that relates $a_{i}$ to $a_{j}$. Then $\Delta=\left\{v_{i} \delta_{i j} v_{j}\right\}_{i, j=1}^{n}$ is a consistent scenario and we call this the scenario (or basic constraint network) induced by $\left(a_{i}\right)_{i=1}^{n}$. 
The consistency of a constraint network can be partially determined by path-consistency algorithms. We say a network $\Gamma=\left\{v_{i} R_{i j} v_{j}\right\}_{i, j=1}^{n}$ is path-consistent if

$$
R_{j i}=R_{i j}^{\sim}, \quad \varnothing \neq R_{i j} \subseteq R_{i k} \circ_{w} R_{k j}
$$

for any $i, j$ and any $k \neq i, j$. In case $\Gamma$ is a basic network, this is equivalent to saying that every subnetwork involving three variables of $\Gamma$ is consistent.

Path-consistency can be enforced in cubic time (Vilain \& Kautz, 1986). That is, if we apply the path-consistency algorithm on a constraint network $\Gamma$, then in cubic time the algorithm will terminate and we either get an empty constraint (and hence know that $\Gamma$ is inconsistent) or transform $\Gamma$ into an equivalent path-consistent network. For basic networks, it is easy to see that consistency implies path-consistency, but the opposite proposition does not always hold.

In the following subsections we recall the qualitative topological and directional calculi that will be discussed in this paper.

\subsection{The Region Connection Calculus RCC8}

The region connection calculus (RCC) (Randell et al., 1992) is a first-order theory based on a binary connectedness relation. Standard RCC models arise from topological spaces. In this paper, we are only concerned with interpretations of RCC in the real plane, which provides arguably the most important model for RCC. Another reason lies in that the directional calculi considered in this paper are also defined over the real plane. A plane region (or region) is a nonempty regular closed subset of the real plane. We only consider bounded regions, as cardinal directions only involve bounded regions. But these regions could have multi-pieces and/or have holes. ${ }^{1}$

One standard interpretation of RCC is based on the Whiteheadean connectedness (Whitehead, 1929) on plane regions, where two regions are connected if they have a common point. This connectedness may be considered too weak in many cases. For example, "a worm cannot pass from the interior of one apple to another, which touch just at a point, without becoming visible to the exterior - so from the worm's point of view we might as well say that the apples are not 'sufficiently' connected." (Borgo, Guarino, \& Masolo, 1996, p. 223) In this paper, we also consider a stronger connectedness, in which two regions are regarded as connected if their intersection is at least onedimensional ( $\mathrm{Li}, \mathrm{Liu}, \&$ Wang, 2013). In the case of a rectangular grid of spatial primitive entities, as already noted, strong and weak connectedness correspond to, respectively, the important notion of 4- and 8-neighbourhood of pixels commonly used in Computer Vision.

In both interpretations, the relations in Table 2 and the converses of TPP and NTPP form a JEPD set. Write $\mathcal{B}_{r c c 8}$ and $\mathcal{B}_{r c c 8^{\prime}}$ for these two sets. We call the Boolean algebras generated by $\mathcal{B}_{r c c 8}$ and $\mathcal{B}_{r c c 8^{\prime}}$, respectively, the weak and the strong RCC8 models, written as RCC8 and RCC $8^{\prime}$. Strong connectedness has been considered by Borgo et al., (1996), Cohn and Varzi (1999), and $\mathrm{Li}$ et al., (2013). It is easy to see that, as relations, strong connectedness is contained in weak connectedness. Table 2 illustrates a configuration (the 2 nd from the left) which is an instance of EC in RCC8 but an instance of $\mathbf{D C}$ in $\mathrm{RCC} 8^{\prime}$, and a configuration (the 2nd from the right) which is an instance of TPP in RCC8 but an instance of NTPP in RCC8'.

1. We stress here that the restriction of RCC to bounded plane regions does not affect the complexity of reasoning with RCC8, as every consistent RCC8 network has a solution in any RCC model (Li, 2006b). 

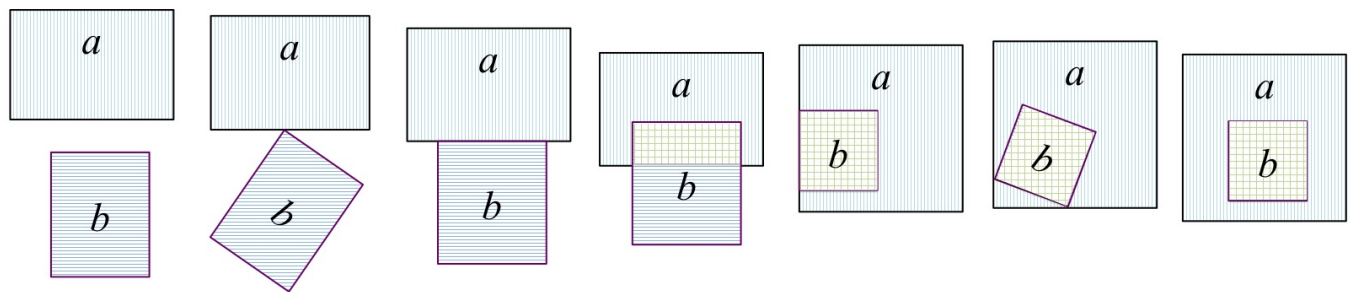

$\begin{array}{lll}\text { RCC8 } & \text { DC } & \text { EC } \\ \text { RCC8' } & \text { DC } & \text { DC }\end{array}$

EC

PO

EC
TPP

TPP

TPP NTPP

NTPP NTPP

\begin{tabular}{c|c|c|c}
\hline Relation & Symb. & Definition (weak) & Definition (strong) \\
\hline equals & EQ & $a=b$ & $a=b$ \\
disconnected & DC & $a \cap b=\varnothing$ & $\operatorname{dim}(a \cap b) \leq 0$ \\
externally connected & EC & $a \cap b \neq \varnothing \wedge a^{\circ} \cap b^{\circ}=\varnothing$ & $\operatorname{dim}(a \cap b)=1$ \\
\hline partially overlap & PO & $a^{\circ} \cap b^{\circ} \neq \varnothing \wedge$ & $a^{\circ} \cap b^{\circ} \neq \varnothing \wedge$ \\
& $a \nsubseteq b \wedge a \nsupseteq b$ & $a \nsubseteq b \wedge a \nsupseteq b$ \\
\hline tangential proper part & TPP & $a \subset b \wedge a \not \subset b^{\circ}$ & $a \subset b \wedge \operatorname{dim}(\partial a \cap \partial b)=1$ \\
non-tangential proper part & NTPP & $a \subset b^{\circ}$ & $a \subset b \wedge \operatorname{dim}(\partial a \cap \partial b) \leq 0$ \\
\hline
\end{tabular}

Table 2: The set of basic RCC8 and RCC $8^{\prime}$ relations, where $a, b$ are two plane regions and $x^{\circ}, \partial x, \operatorname{dim}(x)$ denote, respectively, the interior, boundary, and dimension of $x$. Note that for notational convenience we set $\operatorname{dim}(\varnothing)=-1$.

Remark 1. As far as consistency and realisations are concerned, Li (2006b) has shown that any consistent RCC8 network has a solution in any RCC model. The cubic realisation algorithm described there can be used to construct a solution in both the weak and the strong RCC8 models. This implies in particular that an RCC8 network has a solution in the weak RCC8 model iff it has a solution in the strong RCC8 model. As we will show in this paper, this is, however, not the case when cardinal directions are combined with topological relations.

In the following, we recall some important properties of the three maximal tractable subclasses $\widehat{\mathcal{H}}_{8}, \mathcal{C}_{8}$, and $\mathcal{Q}_{8}$ of RCC8 identified by Renz (1999). A complete list of relations in these subclasses can be found in Appendix A of the work of Renz (2002).

Lemma 2. Suppose $R$ is a non-basic $R C C 8$ relation such that $R \cap\{\mathbf{D C}, \mathbf{E C}, \mathbf{P O}\}=\varnothing$. Then

(1) $R \in \mathcal{Q}_{8}$ iff $R$ is either $\{\mathbf{T P P}, \mathbf{N T P P}\}$ or $\left\{\mathbf{T P P}^{\sim}, \mathbf{N T P P}^{\sim}\right\}$.

(2) $R \in \widehat{\mathcal{H}}_{8}$ iff $R$ is in $\mathcal{Q}_{8}$ or one of the following relations

$$
\{\mathbf{T P P}, \mathbf{E Q}\},\{\mathbf{T P P}, \mathbf{N T P P}, \mathbf{E Q}\},\left\{\mathbf{T P P}^{\sim}, \mathbf{E Q}\right\},\left\{\mathbf{T P P}^{\sim}, \mathbf{N T P P}^{\sim}, \mathbf{E Q}\right\} .
$$

(3) $R \in \mathcal{C}_{8}$ iff $R$ is in $\widehat{\mathcal{H}}_{8}$, or either $\{\mathbf{N T P P}, \mathbf{E Q}\}$ or $\left\{\mathbf{N T P P}^{\sim}, \mathbf{E Q}\right\}$.

We note the above lemma does not define these subclasses. In particular, these subclasses do include RCC8 relations $R$ such that $R \cap\{\mathbf{D C}, \mathbf{E C}, \mathbf{P O}\} \neq \varnothing$.

Renz also shows that a consistent scenario can be constructed in $O\left(n^{2}\right)$ time for any pathconsistent network $\Theta$ over one of the three maximal tractable subclasses. 
Theorem 3 (Renz, 1999). A consistent scenario $\Theta_{s}$ of a path-consistent network $\Theta$ of constraints over $\widehat{\mathcal{H}}_{8}, \mathcal{C}_{8}$, or over $\mathcal{Q}_{8}$ can be computed in $O\left(n^{2}\right)$ time, by replacing every constraint $\left(v_{i} R v_{j}\right) \in \Theta$ with $\left(v_{i}\right.$ base $\left.(R) v_{j}\right) \in \Theta_{s}$, where base $(R)$ is a basic relation obtained as follows:

(1) If $R \in \mathcal{B}$, then base $(R)=R$;

(2) else if $\{\mathbf{D C}\} \subseteq R$, then base $(R)=\{\mathbf{D C}\}$;

(3) else if $\{\mathbf{E C}\} \subseteq R$ and $\mathcal{S}=\mathcal{Q}_{8}$ or $\mathcal{S}=\widehat{\mathcal{H}}_{8}$, then base $(R)=\{\mathbf{E C}\}$;

(4) else if $\{\mathbf{P O}\} \subseteq R$, then base $(R)=\{\mathbf{P O}\}$;

(5) else if $\{\mathbf{N T P P}\} \subseteq R$ and $\mathcal{S}=\mathcal{C}_{8}$, then base $(R)=\{\mathbf{N T P P}\}$;

(6) else if $\{\mathbf{T P P}\} \subseteq R$, then base $(R)=\{\mathbf{T P P}\}$;

(7) else $\operatorname{base}(R)=\operatorname{base}\left(R^{\sim}\right)$.

In what follows, we call $\Theta_{s}$ the canonical consistent scenario of $\Theta$.

\subsubsection{ReAlisation OF BASiC RCC8 NeTwORKS}

It is known that, for basic RCC8 networks, path-consistency implies consistency (Nebel, 1995). We next give a a short description of the cubic realisation algorithm proposed by $\mathrm{Li}$ (2006b), as we need to devise a similar algorithm later for the combination cases.

Given a basic RCC8 network $\Theta=\left\{v_{i} \theta_{i j} v_{j}\right\}_{i, j=1}^{n}$, suppose $\Theta$ is path-consistent. An ntpp-chain in $\Theta$ is defined to be a series of variables $v_{i_{1}}, v_{i_{2}}, \cdots, v_{i_{k}}$ such that $v_{i_{s}} \mathbf{N T P P} v_{i_{s+1}} \in \Theta$ for all $s=1, \cdots, k-1$. The ntpp-level $l(i)$ of a variable $v_{i}$ is defined to be the maximum length of the ntpp-chains contained in $\Theta$ that ends with $v_{i}$.

A realisation can be constructed as follows, where a variable may be interpreted as a bounded region with multiple pieces. Without loss of generality, we assume $\left(v_{i} \mathbf{E} \mathbf{Q} v_{j}\right) \in \Theta$ only when $i=j$. We first define for each variable $v_{i}$ a finite set $X_{i}$ of control points as follows. For each $i$, introduce a point $P_{i}$ to $v_{i}$; if $v_{i} \mathbf{E C} v_{j}$ or $v_{i} \mathbf{P O} v_{j}$, then introduce a point $P_{i j}$ to $v_{i}$; if $v_{i} \mathbf{T P P} v_{j}$ or $v_{i} \mathbf{N T P P} v_{j}$, then put all $X_{i}$ points into $X_{j}$. We then expand each point $P$ in $X_{i}$ a little to obtain a square $s(P)$. These squares are pairwise disjoint. Then, taking the union of these squares, we obtain an instantiation of bounded regions to these $v_{i}$. This works for all but the EC and NTPP constraints. Further modifications are needed to cope with these constraints (cf. Li, 2006b or Appendix C of this paper).

\subsection{Interval Algebra and Rectangle Algebra}

In this subsection, we recall Interval Algebra (IA) (Allen, 1983) and Rectangle Algebra (RA) (Balbiani et al., 1999). IA is the qualitative calculus generated by the 13 basic relations between closed intervals on the real line shown in Table 3 . We write

$$
\mathcal{B}_{\text {int }}=\{\mathrm{b}, \mathrm{m}, \mathrm{o}, \mathrm{s}, \mathrm{d}, \mathrm{f}, \mathrm{eq}, \mathrm{fi}, \mathrm{di}, \mathrm{si}, \mathrm{oi}, \mathrm{mi}, \mathrm{bi}\}
$$

for the set of basic IA relations. Ligozat (1994) defines the dimension ${ }^{2}$ of a basic interval relation as 2 minus the number of equalities appearing in the definition of the relation (see Table 3). That is,

2. We stress that this notion of dimension is different from the topological dimension. 
for basic relations we have

$$
\operatorname{dim}(\mathbf{e q})=0, \operatorname{dim}(\mathbf{m})=\operatorname{dim}(\mathbf{s})=\operatorname{dim}(\mathbf{f})=1, \operatorname{dim}(\mathbf{b})=\operatorname{dim}(\mathbf{o})=\operatorname{dim}(\mathbf{d})=2 .
$$

For a non-basic relation $R$ we define

$$
\operatorname{dim}(R)=\max \{\operatorname{dim}(\theta): \theta \text { is a basic relation in } R\} .
$$

\begin{tabular}{|c|c|c|c|c|}
\hline Relation & Symb. & Conv. & Dim. & Definition \\
\hline before & $\mathrm{b}$ & bi & 2 & $x^{+}<y^{-}$ \\
meets & $\mathrm{m}$ & $\mathrm{mi}$ & 1 & $x^{+}=y^{-}$ \\
overlaps & o & oi & 2 & $x^{-}<y^{-}<x^{+}<y^{+}$ \\
starts & $\mathrm{s}$ & $\mathrm{si}$ & 1 & $x^{-}=y^{-}<x^{+}<y^{+}$ \\
during & $\mathrm{d}$ & $\mathrm{di}$ & 2 & $y^{-}<x^{-}<x^{+}<y^{+}$ \\
finishes & $\mathrm{f}$ & $\mathrm{fi}$ & 1 & $y^{-}<x^{-}<x^{+}=y^{+}$ \\
equals & eq & eq & 0 & $x^{-}=y^{-}<x^{+}=y^{+}$ \\
\hline
\end{tabular}

(i)

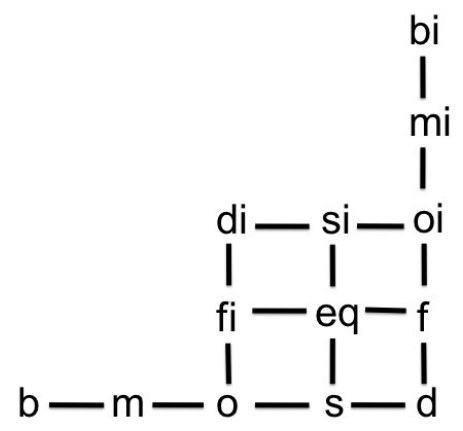

(ii)

Table 3: IA basic relations (i) definitions and (ii) conceptual neighbourhood graph, where $x=$ $\left[x^{-}, x^{+}\right], y=\left[y^{-}, y^{+}\right]$are two intervals.

Nebel and Bürckert (1995) have shown that there is a unique maximal tractable subclass of IA which contains all basic relations. This subclass, written as $\mathcal{H}$, is known as the ORD-Horn class. Using the conceptual neighbourhood graph (CNG) of IA (Freksa, 1992), Ligozat (1994) gives a geometrical characterisation for ORD-Horn relations. Consider the CNG of IA (shown in Table 3 (ii)) as a partially ordered set $\left(\mathcal{B}_{\text {int }}, \preceq\right)$ (by interpreting any relation smaller than its right or upper neighbours). For $\theta_{1}, \theta_{2} \in \mathcal{B}_{\text {int }}$ with $\theta_{1} \preceq \theta_{2}$, we write $\left[\theta_{1}, \theta_{2}\right]$ as the set of basic interval relations $\theta$ such that $\theta_{1} \preceq \theta \preceq \theta_{2}$, and call such a relation a convex interval relation. An IA relation $R$ is called pre-convex if it can be obtained from a convex relation by removing one or more basic relations with dimension lower than $R$. For example, $[\mathrm{o}, \mathrm{eq}]=\{\mathrm{o}, \mathrm{s}, \mathrm{fi}, \mathrm{eq}\}$ is a convex relation and $\{\mathrm{o}, \mathrm{eq}\}$ is a pre-convex relation. Ligozat has shown that ORD-Horn relations are precisely pre-convex relations.

Nebel and Bürckert also show that every path-consistent IA network over $\mathcal{H}$ is consistent. Furthermore, we can construct a consistent scenario for every path-consistent IA network over $\mathcal{H}$ in quadratic time.

Suppose $R$ is an IA relation and let

$$
R^{\text {core }}=\left\{\theta \in \mathcal{B}_{\text {int }}: \operatorname{dim}(\theta)=\operatorname{dim}(R), \theta \in R\right\} .
$$

By (6) it is clear that $R^{\text {core }}=\{\mathrm{eq}\}$ iff $R=\{\mathrm{eq}\}$, and $R^{\text {core }}=R \cap\{\mathrm{b}, \mathrm{o}, \mathrm{d}$, di, oi, bi $\}$ if $R \cap$ $\{\mathrm{b}, \mathrm{o}, \mathrm{d}, \mathrm{di}, \mathrm{oi}, \mathrm{bi}\}$ is nonempty, and $R^{\text {core }}=R \backslash\{\mathrm{eq}\}$ otherwise. Then we have

Lemma 4. (Renz, 1999) Suppose $\Theta=\left\{v_{i} R_{i j} v_{j}\right\}_{i, j=1}^{n}$ is a path-consistent IA network over $\mathcal{H}$. Let

$$
\Theta^{\text {core }}=\left\{v_{i} R_{i j}^{\text {core }} v_{j}\right\}_{i, j=1}^{n} \text {. }
$$

Then $\Theta^{\text {core }}$ is also path-consistent. 
From Table 3 (ii) it is easy to see that a pre-convex relation $R$ has dimension 1 iff either $R$ is a 1-dim basic relation or $R$ is contained in $\{\mathrm{s}, \mathrm{eq}, \mathrm{si}\}$ or $\{\mathrm{fi}, \mathrm{eq}, \mathrm{f}\}$. As a consequence, we know

Corollary 5. Suppose $\Theta=\left\{v_{i} R_{i j} v_{j}\right\}_{i, j=1}^{n}$ is a path-consistent network over $\mathcal{H}$. Then $\Theta$ has a consistent scenario $\Theta^{*}=\left\{v_{i} R_{i j}^{*} v_{j}\right\}_{i, j=1}^{n}$ which has the following property:

$\operatorname{dim}\left(R_{i j}^{*}\right)=\operatorname{dim}\left(R_{i j}\right)$, and

$R_{i j}^{*}=\{$ eq $\}$ only if $R_{i j}=\{$ eq $\}, R_{i j}^{*}=\{m\}$ only if $R_{i j}=\{m\}, R_{i j}^{*}=\{m i\}$ only if $R_{i j}=\{m i\}$.

This result shows that, for any path-consistent network $\Theta$ over $\mathcal{H}$, we can construct in quadratic time a consistent scenario for $\Theta$.

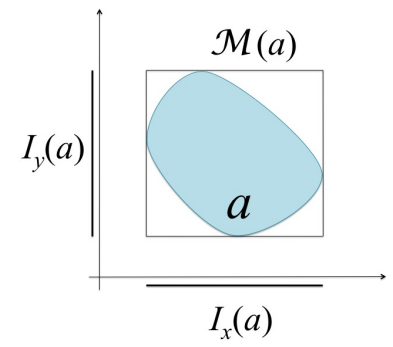

(i)

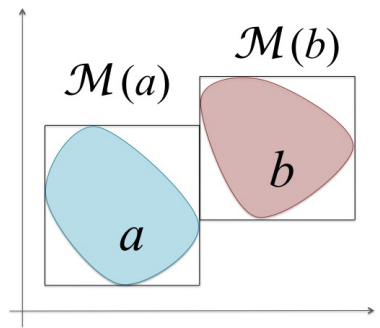

(ii)

Figure 2: (i) The minimum bounding rectangle $\mathcal{M}(a)$ of a region $a$; (ii) the RA relation of $a$ to $b$ is $\mathrm{m} \otimes 0$.

IA can be naturally extended to regions in the plane. We assume an orthogonal basis in the Euclidean plane. For a bounded region $a$, its minimum bounding rectangle (MBR), denoted by $\mathcal{M}(a)$, is the smallest rectangle which contains $a$ and whose sides are parallel to the axes of the basis. We write $I_{x}(a)$ and $I_{y}(a)$ as, respectively, the $x$ - and $y$-projections of $\mathcal{M}(a)$. The basic rectangle relation between two bounded regions $a, b$ is $\alpha \otimes \beta$ iff $\left(I_{x}(a), I_{x}(b)\right) \in \alpha$ and $\left(I_{y}(a), I_{y}(b)\right) \in \beta$, where $\alpha, \beta$ are two basic IA relations (see Figure 2 for illustration). We write $\mathcal{B}_{\text {rec }}$ for the set of basic rectangle relations, i.e.,

$$
\mathcal{B}_{\text {rec }}=\left\{\alpha \otimes \beta: \alpha, \beta \in \mathcal{B}_{\text {int }}\right\} .
$$

There are 169 different basic rectangle relations in $\mathcal{B}_{\text {rec }}$. The Rectangle Algebra (RA) is the algebra generated by relations in $\mathcal{B}_{\text {rec }}$ (Balbiani et al., 1999).

The following definitions will be used later.

Definition 6. Suppose $\alpha=\rho_{1} \otimes \rho_{2}$ is a basic RA relation. We say $\alpha$ is a 0 -meet relation if $\rho_{1}, \rho_{2} \in\{\mathrm{m}, \mathrm{mi}\}$, and is a corner relation if $\rho_{1}, \rho_{2} \in\{\mathrm{m}, \mathrm{mi}, \mathrm{s}, \mathrm{si}, \mathrm{f}, \mathrm{fi}, \mathrm{eq}\}$. In general, we say a non-basic RA relation $R=\left\{\alpha_{1}, \ldots, \alpha_{k}\right\}(k \geq 2)$ is a corner relation if each $\alpha_{i}(1 \leq i \leq k)$ is a corner relation.

By definition, each 0-meet relation is a corner relation. Furthermore, it is easy to see that a basic RA relation $\alpha$ is a 0-meet relation iff, for every two rectangles $r, r^{\prime}$ with $\left(r, r^{\prime}\right) \in \alpha, r \cap r^{\prime}$ is a singleton in the plane; and $\alpha$ is a corner relation iff every two rectangles $r, r^{\prime}$ with $\left(r, r^{\prime}\right) \in \alpha$ have, at least, a corner point in common.

The following lemma is straightforward. 
Lemma 7. Let $\Delta=\left\{v_{i}\left(R_{i j} \otimes S_{i j}\right) v_{j}\right\}_{i, j=1}^{n}$ be an $R A$ network, where $R_{i j}$ and $S_{i j}$ are arbitrary IA relations. Then $\Delta$ is satisfiable iff its projections $\Delta^{x}=\left\{x_{i} R_{i j} x_{j}\right\}_{i, j=1}^{n}$ and $\Delta^{y}=\left\{y_{i} S_{i j} y_{j}\right\}_{i, j=1}^{n}$ are satisfiable IA networks.

By Corollary 5 and the above lemma we have

Lemma 8. Suppose $\Delta=\left\{v_{i} R_{i j} v_{j}\right\}$ is a path-consistent $R A$ network over $\mathcal{H} \times \mathcal{H}$. Then $\Delta$ has a consistent scenario $\Delta^{*}=\left\{v_{i} \delta_{i j} v_{j}\right\}$ such that

- $\delta_{i j}$ is a 0 -meet relation iff $R_{i j}$ is a 0 -meet basic relation, and

- $\delta_{i j}$ is a corner relation iff $R_{i j}$ consists only of basic corner relations.

As a consequence, we know $\mathcal{H} \times \mathcal{H}$ is a tractable subclass of RA. No maximal tractable subclass has been identified for RA, but a larger tractable subclass of RA has been identified (Balbiani et al., 1999).

We next show that each path-consistent basic IA or RA network has a canonical solution in the following sense.

Definition 9 (canonical tuple of intervals (rectangles)). Suppose $\mathfrak{m}=\left(\left[m_{i}^{-}, m_{i}^{+}\right]\right)_{i=1}^{n}$ is an $n$-tuple of intervals. Let $E(\mathfrak{m})$ be the set of the values of the end points of intervals in $\mathfrak{m}$. We say $\mathfrak{m}$ is canonical iff $E(\mathfrak{m})=\{0,1, \cdots, M\}$. A tuple of rectangles $\left(m_{i}\right)_{i=1}^{n}$ is canonical iff its $x$ - and $y$-projections, $\left(I_{x}\left(m_{i}\right)\right)_{i=1}^{n}$ and $\left(I_{y}\left(m_{i}\right)\right)_{i=1}^{n}$, are canonical tuples of intervals. A solution of an IA (RA, respectively) network is called a canonical solution if it is a canonical tuple of intervals (rectangles, respectively).

For a basic satisfiable IA network, we can compute the total order of all the end points. Hence we can obtain a canonical solution (by assigning 0 to the first end point, 1 to the second, etc.). This gives us the following proposition.

Proposition 10. Suppose $\Theta$ is a satisfiable basic IA (RA) constraint network. Then $\Theta$ has a unique canonical solution.

\subsection{Cardinal Direction Calculus}

The cardinal direction calculus (CDC) was proposed by Goyal and Egenhofer (1997). Given a bounded region $b$ in the real plane, by extending the four edges of $\mathcal{M}(b)$, we partition the plane into nine tiles, denoted by $b^{i j}(1 \leq i, j \leq 3)$, see Figure 3 (i) for illustration.

For a primary region $a$ and a reference region $b$, the CDC relation of $a$ to $b$, denoted by $\delta_{a b}$, is encoded in a $3 \times 3$ Boolean matrix $\left(d_{i j}\right)_{1 \leq i, j \leq 3}$, where $d_{i j}=1$ iff $a^{\circ} \cap b^{i j} \neq \varnothing$ (where $a^{\circ}$ is again the interior of $a$ ). For example, the basic CDC relations $\delta_{a b}$ and $\delta_{b a}$ for the regions $a, b$ in Figure 3(ii) are represented by the following matrices.

$$
\delta^{*}=\delta_{a b}=\left[\begin{array}{ccc}
0 & 0 & 0 \\
1 & 0 & 0 \\
0 & 0 & 0
\end{array}\right], \quad \gamma^{*}=\delta_{b a}=\left[\begin{array}{ccc}
0 & 0 & 1 \\
0 & 0 & 1 \\
0 & 0 & 1
\end{array}\right]
$$

A CDC relation can be any but the zero Boolean matrix, so there are $2^{9}-1=511$ basic relations in CDC. We denote this set by $\mathcal{B}_{c d c}$. A pair of basic CDC relations $(\delta, \gamma)$ is called a consistent pair 


\begin{tabular}{|c|c|c|}
\hline$b^{11}$ & $b^{12}$ & $b^{13}$ \\
\hline$b^{21}$ & $b$ & $b^{23}$ \\
\hline$b^{31}$ & $b^{32}$ & $b^{33}$ \\
\hline
\end{tabular}

(i)

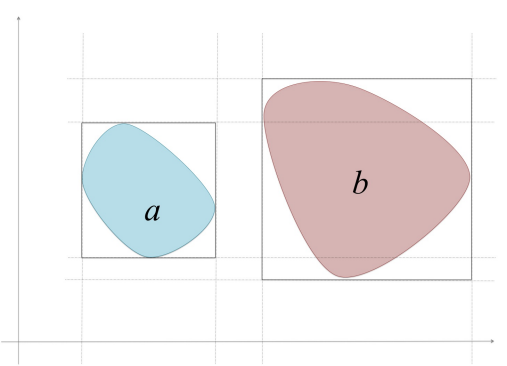

(ii)

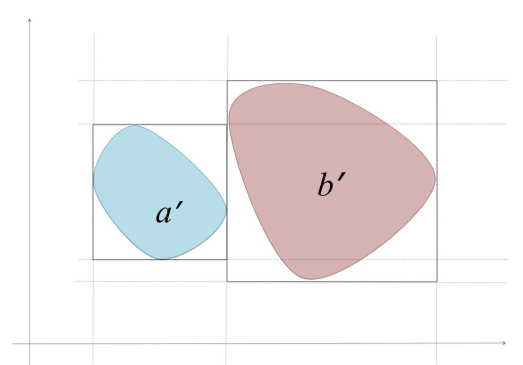

(iii)

Figure 3: Illustrations of (i) the nine tiles of a reference region; (ii) and (iii): two solutions of the CDC basic constraint network $\left\{v_{1} \delta^{*} v_{2}, v_{2} \gamma^{*} v_{1}\right\}$, where $\delta^{*}$ and $\gamma^{*}$ are defined in Eq. (9).

if the constraint network $\left\{v_{1} \delta v_{2}, v_{2} \gamma v_{1}\right\}$ has a solution. We also call $\gamma$ a weak converse of $\delta$ if $(\delta, \gamma)$ is a consistent pair. Figure 4 shows that a basic CDC relation may have more than one weak converse. Therefore, we need both the relation of $a$ to $b$ and the relation of $b$ to $a$ to give a complete description (in terms of the CDC calculus) of the directional information between two regions $a, b$.

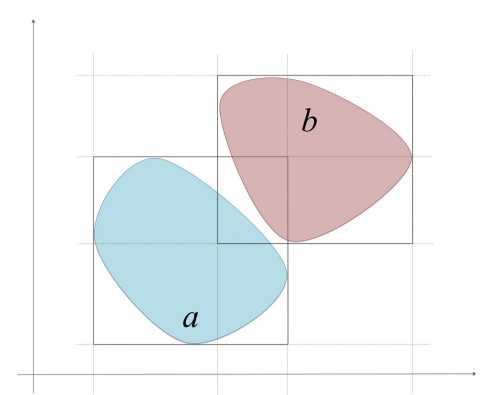

(i)

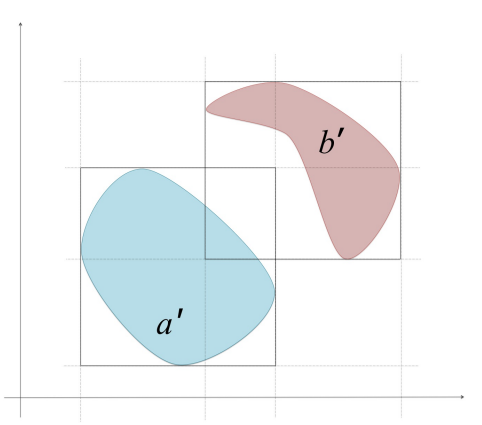

(ii)

Figure 4: Illustration of two consistent CDC pairs (i) $\left(\delta_{a b}, \delta_{b a}\right)$ and (ii) $\left(\delta_{a^{\prime} b^{\prime}}, \delta_{b^{\prime} a}\right)$, where $\delta_{a b}=$ $\delta_{a^{\prime} b^{\prime}}$ but $\delta_{b a} \neq \delta_{b^{\prime} a^{\prime}}$. Also note that the rectangle relation between $a, b$ and that between $a^{\prime}, b^{\prime}$ are both $0 \otimes 0$.

In the following we show that there is a strong connection between CDC and RA relations.

Definition 11. (Zhang et al., 2008; Liu et al., 2010) For a pair of basic CDC relations $(\delta, \gamma)$, we define the $x$-projective interval relation of $(\delta, \gamma)$, written as $\iota^{x}(\delta, \gamma)$, as the disjunction of all basic IA relations $\alpha$ which has an instance that is the $x$-projection of some solution of $\left\{v_{1} \delta v_{2}, v_{2} \gamma v_{1}\right\}$, i.e.

$$
\iota^{x}(\delta, \gamma)=\left\{\alpha \in \mathcal{B}_{\text {int }}:\left(\exists m_{1}, m_{2}\right)\left[\left(m_{1}, m_{2}\right) \in \delta \wedge\left(m_{2}, m_{1}\right) \in \gamma \wedge\left(I_{x}\left(m_{1}\right), I_{x}\left(m_{2}\right)\right) \in \alpha\right]\right\} .
$$

A similar definition applies for the $y$-direction.

Note that if $(\delta, \gamma)$ is not a consistent pair, then both $\iota^{x}(\delta, \gamma)$ and $\iota^{y}(\delta, \gamma)$ are the empty relation. If $(\delta, \gamma)$ is a consistent pair, then we can prove (Liu et al., 2010) that its $x$ - (or $y$-) projective interval 
relation is an IA relation $R$ which has the following property

$$
R=\{\mathrm{b}, \mathrm{m}\} \text { or } R=\{\mathrm{bi}, \mathrm{mi}\} \text {, or } R \text { is a basic IA relation in }\{\mathrm{o}, \mathrm{s}, \mathrm{d}, \mathrm{f}, \mathrm{eq}, \mathrm{oi}, \mathrm{si}, \mathrm{di}, \mathrm{fi}\} .
$$

The two projective interval relations can then be combined into an RA relation.

Definition 12. (Zhang et al., 2008; Liu et al., 2010) For a pair of basic CDC relations $(\delta, \gamma)$, we call $\iota(\delta, \gamma)=\iota^{x}(\delta, \gamma) \otimes \iota^{y}(\delta, \gamma)$ the RA relation induced by $(\delta, \gamma)$. In general, for a basic CDC constraint network $\Delta=\left\{v_{i} \delta_{i j} v_{j}\right\}_{i, j=1}^{n}$, we call $\iota(\Delta)=\left\{v_{i} R_{i j} v_{j}\right\}_{i, j=1}^{n}$ the RA constraint network induced by $\Delta$, where $R_{i j}=\iota\left(\delta_{i j}, \delta_{j i}\right)$.

Note that $\iota(\delta, \gamma)$ is not necessarily a basic RA relation. If $(\delta, \gamma)$ is consistent, then we know the RA relation $\iota(\delta, \gamma)$ has the form $\alpha \otimes \beta$, where $\alpha, \beta$ are IA relations that satisfy (10). Furthermore, a solution of $\left\{v_{1} \delta v_{2}, v_{2} \gamma v_{1}\right\}$ is always a solution of $\left\{v_{1} \iota(\delta, \gamma) v_{2}\right\}$. We note that a solution of $\left\{v_{1} \iota(\delta, \gamma) v_{2}\right\}$ is not necessarily a solution of $\left\{v_{1} \delta v_{2}, v_{2} \gamma v_{1}\right\}$.

Take the consistent pair $\left(\delta^{*}, \gamma^{*}\right)$ defined in (9) as an example. Figure 3 (ii) and (iii) show two solutions $(a, b)$ and $\left(a^{\prime}, b^{\prime}\right)$ of the basic CDC constraint network $\left\{v_{1} \delta^{*} v_{2}, v_{2} \gamma^{*} v_{1}\right\}$. This implies by definition that $\iota^{x}\left(\delta^{*}, \gamma^{*}\right)$ contains $\{\mathrm{b}, \mathrm{m}\}$. It is easy to see from the definition that $\iota^{x}\left(\delta^{*}, \gamma^{*}\right)$ contains no other basic IA relations and $\iota^{x}\left(\delta^{*}, \gamma^{*}\right)=\{\mathrm{b}, \mathrm{m}\}$. Similarly, we can show $\iota^{y}\left(\delta^{*}, \gamma^{*}\right)=$ $\{\mathrm{d}\}$. This shows that this consistent pair $\left(\delta^{*}, \gamma^{*}\right)$ corresponds to basic RA relations, viz. $\mathrm{m} \otimes \mathrm{d}$ and $b \otimes d$.

\subsubsection{Canonical Solutions of Basic CDC Networks}

Just like IA and RA, consistent CDC networks also have 'canonical' solutions.

Definition 13 (regular solution, Zhang et al., 2008; Liu et al., 2010). Suppose $\mathfrak{m}=\left(m_{i}\right)_{i=1}^{n}$ is a solution of a basic CDC constraint network $\Delta$. We say that $\mathfrak{m}$ is maximal if $m_{i}^{\prime} \subseteq m_{i}$ holds for any solution $\left(m_{i}^{\prime}\right)_{i=1}^{n}$ of $\Delta$ with $\mathcal{M}\left(m_{i}\right)=\mathcal{M}\left(m_{i}^{\prime}\right)$; we say $\mathfrak{m}$ is regular if $\mathfrak{m}$ is maximal and $\left(\mathcal{M}\left(m_{i}\right)\right)_{i=1}^{n}$ is a canonical tuple of rectangles.

A basic CDC network in general has many regular solutions, but we have the following result.

Proposition 14. Let $\Delta$ be a basic CDC network. Suppose $\Gamma$ is a basic RA network that refines $\iota(\Delta)$, the induced RA network of $\Delta$. Then we can determine in cubic time whether $\Delta$ has a solution that also satisfies $\Gamma$. Moreover, if $\Delta$ has a solution, then it has a unique regular solution which also satisfies $\Gamma$. Furthermore, this unique regular solution can be constructed in cubic time.

Proof. The proof is similar to that for Proposition 12 in the work of Liu et al., (2010). A sketch is given in Appendix A.

From the proof of the above result, we can see that each region $m_{i}$ in a regular solution $\left(m_{i}\right)_{i=1}^{n}$ consists of unit cells (i.e. rectangles of the form $[i, i+1] \times[j, j+1]$, where $i, j \in \mathbb{Z}$ ) in the canonical solution of $\Gamma$, i.e. for each region $m_{i}$ and each cell $c$, we have either $c \subseteq m_{i}$ or $c \cap m_{i}{ }^{\circ}=\varnothing$.

For a basic CDC network $\Delta$, there may exist exponentially many different basic RA networks that refine $\iota(\Delta)$. Hence, $\Delta$ may have exponentially many different regular solutions (see Figure 11(a) for an example of such a network). However, to verify that $\Delta$ has a solution, we need only prove that $\Delta$ has a solution for some special basic RA network that refines $\iota(\Delta)$ (Liu et al., 2010, Proposition 12). ${ }^{3}$ Therefore, the consistency of $\Delta$ can be determined in cubic time, and, if $\Delta$ is consistent, a regular solution can be constructed in cubic time (Liu et al., 2010).

3. Such a special network is called a "meet-free" basic RA network in the work of Liu et al., (2010). 


\section{The Joint Satisfaction Problem}

After the preparatory introduction of basic notions and essential results of qualitative calculi, we are now ready to describe the joint satisfaction problem.

Let $\mathbf{M}_{1}$ and $\mathbf{M}_{2}$ be two qualitative calculi over the same domain $\mathbb{U}$. Suppose $\mathcal{S}_{i}$ is a subclass of $\mathbf{M}_{i}(i=1,2)$. We write $\mathbf{J S P}\left(\mathcal{S}_{1}, \mathcal{S}_{2}\right)$ for the joint satisfaction problem (Gerevini \& Renz, 2002; Li, 2007) over $\mathcal{S}_{1}$ and $\mathcal{S}_{2}$.

Suppose $\Theta=\left\{v_{i} T_{i j} v_{j}\right\}_{i, j=1}^{n}$ is a constraint network over $\mathcal{S}_{1}$, and $\Delta=\left\{v_{i} D_{i j} v_{j}\right\}_{i, j=1}^{n}$ is a constraint network over $\mathcal{S}_{2}$ involving the same variables. Then we say $\Theta \uplus \Delta$ is an instance of $\operatorname{JSP}\left(\mathcal{S}_{1}, \mathcal{S}_{2}\right)$. The joint satisfaction problem was first considered for RCC8 and the qualitative size calculus (identical to the Point Algebra in Vilain \& Kautz, 1986) by Gerevini and Renz (2002). Moreover, it was shown that the consistency of a joint network can be approximated by the polynomial bipath-consistency algorithm. Li and Cohn (2012) recently showed that bipath-consistency can be equivalently expressed as below.

Definition 15. Let $\Theta \uplus \Delta$ be a joint constraint network over $\mathbf{M}_{1}$ and $\mathbf{M}_{2}$, where $\Theta=\left\{v_{i} T_{i j} v_{j}\right\}_{i, j=1}^{n}$ and $\Delta=\left\{v_{i} D_{i j} v_{j}\right\}_{i, j=1}^{n}$. We say $\Theta \uplus \Delta$ is bi-closed if $\alpha \cap D_{i j}$ and $T_{i j} \cap \beta$ are nonempty for any basic relation $\alpha \in T_{i j}$, any basic relation $\beta \in D_{i j}$, and any $1 \leq i, j \leq n$ (here we regard each relation as a subset of $\mathbb{U} \times \mathbb{U}$ ). A bi-closed joint network $\Theta \uplus \Delta$ is bipath-consistent if $\Theta$ and $\Delta$ are both path-consistent.

Informally speaking, a joint constraint network is bi-closed if each basic relation of a given relation in one of the calculi is consistent with the corresponding relation in the other calculus.

As a simple example of the joint satisfaction problem, we consider the combination of RA and $\mathrm{CDC}$ in the next subsection.

\subsection{The Combination of RA and CDC}

Let $R$ be a basic CDC relation. Then $R^{\sim}$, the set-theoretic converse (or inverse) relation of $R$ (cf. (1)), may be not representable in the relation algebra CDC (Cicerone \& Di Felice, 2004; Liu et al., 2010). That is, $R^{\sim}$ cannot be represented as the union of several basic CDC relations. In this sense, we say the CDC is not closed under converse. Recently, Schneider et al. (2012) proposed a variant of CDC, called the Object Interaction Model (OIM), which is closed under converse.

For two bounded regions $a, b$, OIM divides the plane into up to $\left(l_{1}+2\right) \times\left(l_{2}+2\right)$ tiles by extending the edges of $\mathcal{M}(a)$ and $\mathcal{M}(b)$, where $l_{1}+1$ and $l_{2}+1$ are the numbers of horizontal and, respectively, vertical lines. It is clear that $1 \leq l_{1}, l_{2} \leq 3$ since edges of $\mathcal{M}(a)$ and $\mathcal{M}(b)$ may coincide. The OIM relation $\phi_{a b}$ is represented by an $l_{1} \times l_{2}$ matrix (also written as $\phi_{a b}$ ) considering existence of interior points of $a$ and/or $b$ in corresponding bounded tiles. Let $\mathrm{T}$ be such a bounded tile. We set the entry corresponding to $\mathrm{T}$ in the matrix $\phi_{a b}$ to 0 if $\mathrm{T}$ has no interior point which is in either $a$ or $b$; and set it 1 (2, respectively) if T has an interior point which is in $a$ ( $b$, respectively) and has no interior point which is in $b$ ( $a$, respectively); and set it 3 otherwise. The converse relation of a basic OIM relation is also a basic OIM relation. In particular, the basic OIM relation $\phi_{b a}$ of $b$ to $a$ can be obtained by swapping the occurrences of 1 and 2 in $\phi_{a b}$.

For example, the OIM relations between the regions in Figure 3 (ii) and (iii) are respectively

$$
\phi_{a b}=\left[\begin{array}{lll}
0 & 0 & 2 \\
1 & 0 & 2 \\
0 & 0 & 2
\end{array}\right], \quad \phi_{a^{\prime} b^{\prime}}=\left[\begin{array}{ll}
0 & 2 \\
1 & 2 \\
0 & 2
\end{array}\right],
$$


and the OIM relations between the regions in Figure 4 are respectively

$$
\phi_{a b}=\left[\begin{array}{lll}
0 & 2 & 2 \\
1 & 3 & 2 \\
1 & 1 & 0
\end{array}\right], \quad \phi_{a^{\prime} b^{\prime}}=\left[\begin{array}{lll}
0 & 2 & 2 \\
1 & 1 & 2 \\
1 & 1 & 0
\end{array}\right] .
$$

We note that for regions $a, b, a^{\prime}, b^{\prime}$ in both figures we have $\delta_{a b}=\delta_{a^{\prime} b^{\prime}}$. This suggests that OIM is finer grained than CDC in the sense that it splits one basic CDC relation into several OIM relations. Nevertheless, since CDC is not closed under converse, we need to consider consistent pairs of basic CDC relations in order to evaluate its expressivity. When comparing the expressivity of the two calculi in this way, we can see that $\left(\delta_{a b}, \delta_{b a}\right) \neq\left(\delta_{a^{\prime} b^{\prime}}, \delta_{b^{\prime} a^{\prime}}\right)$ in Figure 4, but $\left(\delta_{a b}, \delta_{b a}\right)=\left(\delta_{a^{\prime} b^{\prime}}, \delta_{b^{\prime} a^{\prime}}\right)$ in Figure 3. This shows that OIM makes finer distinctions than CDC in describing the scenarios given in Figure 3(ii) and (iii): when saying $a$ is west of $b, \mathrm{CDC}$ does not differentiate if the east boundary of $a$ meets or precedes the west boundary of $b$. The following result shows that OIM is only finer than CDC in describing these cardinal relations, and it is in essence the combination of CDC and RA.

Theorem 16. ( $\mathrm{Li} \& \mathrm{Liu}, 2014$ ) For any two regions $a$ and $b$, we can compute the $R A$ relation of a to $b$, the CDC relation of a to $b$, and the CDC relation of $b$ to a from the OIM relation of $a$ to $b$, and vice versa.

In other words, for each basic OIM relation $\theta$, there exist two basic CDC relations $\delta, \delta^{\prime}$ and a basic RA relation $\gamma$ such that $\theta=\delta \cap \delta^{\prime \sim} \cap \gamma$, i.e. for any two regions $a$ and $b$, the relation $\theta$ is the OIM relation of $a$ to $b$ iff $\delta, \delta^{\prime}$ and $\gamma$ are, respectively, the CDC relation of $a$ to $b$, the CDC relation of $b$ to $a$, and the RA relation of $a$ to $b$. Because basic CDC and RA relations are both JEPD, the above choices of $\delta, \delta^{\prime}, \gamma$ are unique. In the following, we call $\delta$ the CDC relation induced by $\theta$ and call $\gamma$ the RA relation induced by $\theta$. Note that in this case $\delta^{\prime}$ (as a relation of $b$ to $a$ ) is the CDC relation induced by $\theta^{\sim}$, which is the OIM relation of $b$ to $a$.

As a consequence, we have the following result.

Proposition 17. Suppose $\Theta=\left\{v_{i} \theta_{i j} v_{j}\right\}_{i, j=1}^{n}$ is a basic OIM network such that $\theta_{j i}=\theta_{i j}^{\sim}$ for any $i, j$. Let $\Delta=\left\{v_{i} \delta_{i j} v_{j}\right\}_{i, j=1}^{n}$ and $\Gamma=\left\{v_{i} \gamma_{i j} v_{j}\right\}_{i, j=1}^{n}$, where $\delta_{i j}$ and $\gamma_{i j}$ are, respectively, the $C D C$ relation and the RA relation induced by $\theta_{i j}$. Then $\Theta$ is consistent iff the joint network $\Delta \uplus \Gamma$ is consistent.

Proof. Recall that the converse of a basic OIM relation is also a basic OIM relation. Because $\theta_{j i}=\theta_{i j}^{\sim}$, it is straightforward to show that $\theta_{i j}=\delta_{i j} \cap \delta_{j i}^{\sim} \cap \gamma_{i j}$. Therefore, solutions of $\Theta$ are exactly the solutions of $\Delta \uplus \Gamma$.

As a consequence of Propositions 14 and 17 we have

Corollary 18. Let $\Theta, \Delta$ and $\Gamma$ be as given in Proposition 17. Then $\Gamma$ is a basic RA network that refines $\iota(\Delta)$, and the consistency of $\Theta$ can be determined in cubic time. Moreover, if $\Theta$ is consistent, then there is a unique regular solution of $\Delta$ that is also a solution of $\Theta$.

So far, we have described by an example what is a JSP. In the next three sections, we will consider the main task of this paper: the JSP of topological and directional constraints. 


\section{Combination of Weak RCC8 and RA Networks}

In this section we represent topological information as weak RCC8 relations and directional information as RA relations. We first consider the interaction between weak RCC8 and RA relations, then consider the JSP for basic constraints, and, lastly, consider the JSP in general.

\subsection{Interaction Between Weak RCC8 and RA Relations}

Relations in different calculi may interact in the sense that a relation from one calculus may intersect with several relations from the second calculus. We here recall related definitions and preliminary results obtained by Li and Cohn (2012).

Definition 19. Let $T$ be an RCC8 relation and $D$ an RA relation. The RA relation induced by $T$ and the RCC 8 relation induced by $D$ are defined as

$$
\begin{aligned}
R A(T) & =\{\delta: \delta \text { is a basic RA relation and } \delta \cap T \neq \varnothing\} \\
R C C 8(D) & =\{\theta: \theta \text { is a basic RCC8 relation and } \theta \cap D \neq \varnothing\} .
\end{aligned}
$$

Note that a joint network $\Theta \uplus \Delta$ is bi-closed if $\delta_{i j} \subseteq R A\left(\theta_{i j}\right)$ and $\theta_{i j} \subseteq R C C 8\left(\delta_{i j}\right)$ for any $i, j$.

It is easy to see (cf. Li and Cohn, 2012) that $R A(T)=\bigcup\{R A(\{\theta\}): \theta \in T\}$ and

$$
\begin{aligned}
R A(\{\mathbf{D C}\}) \supset R A(\{\mathbf{E C}\}) \supset R A(\{\mathbf{P O}\}) \supset R A(\{\mathbf{T P P}\}) \supset R A(\{\mathbf{N T P P}, \mathbf{E Q}\}), \\
R A(\{\mathbf{P O}\}) \supset R A\left(\left\{\mathbf{T P P} \mathbf{P}^{\sim}\right\}\right) \supset R A(\{\mathbf{N T P P} \sim, \mathbf{E Q}\}),
\end{aligned}
$$

where, for example, $R A(\{\mathbf{E C}\}) \supset R A(\{\mathbf{P O}\})$ holds because, for each basic RA relation $\delta, \delta$ is in $R A(\{\mathbf{E C}\})$ if $\mathcal{M}(a) \cap \mathcal{M}(b) \neq \varnothing$ for all $(a, b) \in \delta$, and $\delta$ is in $R A(\{\mathbf{P O}\})$ if $\mathcal{M}(a) \cap \mathcal{M}(b)$ is a non-degenerate rectangle for all $(a, b) \in \delta$.

Lemma 20. Let $T$ be an $R C C 8$ relation and $D$ an $R A$ relation. Then $R C C 8(D)$ is a relation in the intersection of $\widehat{\mathcal{H}}_{8}, \mathcal{Q}_{8}$, and $\mathcal{C}_{8}$; and $R A(T)$ is a relation in $\mathcal{H} \times \mathcal{H}$ if $T$ is a relation in $\widehat{\mathcal{H}}_{8}$ or $\mathcal{Q}_{8}$.

Proof. This follows from the definitions of $R C C 8(D)$ and $R A(T)$ and a simple table look-up from Appendix A of the work of Renz (2002).

The second statement does not apply to relations in $\mathcal{C}_{8}$. For example, consider $T=\{\mathbf{N T P P}$, EQ $\}$. Then $T$ is a relation in $\mathcal{C}_{8}$, but $R A(T)=\{\mathbf{d} \otimes \mathbf{d}$, eq $\otimes$ eq $\}$ is outside $\mathcal{H} \times \mathcal{H}$.

\subsection{Combination of Basic Networks}

We now consider the combination of RCC8 and RA. First we show that bipath-consistency is not sufficient for consistency in $\mathbf{J S P}\left(\mathcal{B}_{r c c 8}, \mathcal{B}_{r e c}\right)$ (Li \& Cohn, 2012). Let $\Gamma=\left\{v_{i} \gamma_{i j} v_{j}\right\}_{i, j=1}^{4}$ be the basic RA network induced by the four rectangles $m_{i}^{\Gamma}(i=1,2,3,4)$ illustrated below.

Let $\Theta=\left\{v_{i} \theta_{i j} v_{j}\right\}_{i, j=1}^{4}$ be the basic RCC8 constraint network in which $\theta_{12}=\theta_{34}=\{\mathbf{E C}\}$ and all the others are $\{\mathbf{D C}\}$. Clearly, $\Theta$ is satisfiable. Although $\Theta \uplus \Gamma$ is bipath-consistent, it is not satisfiable. This is because, otherwise, there exists a solution $\mathfrak{m}=\left(m_{i}\right)_{i=1}^{4}$ and $\mathcal{M}\left(m_{1}\right) \cap \mathcal{M}\left(m_{2}\right)=$ $\mathcal{M}\left(m_{3}\right) \cap \mathcal{M}\left(m_{4}\right)=\{P\}$ is a singleton. By $\theta_{12}=\theta_{34}=\{\mathbf{E C}\}$ we know $P \in m_{i}(i=1,2,3,4)$. This contradicts $\theta_{13}=\{\mathbf{D C}\}$.

We call point $P$ in the above configuration a conflict point. In general, we have the following definition. 


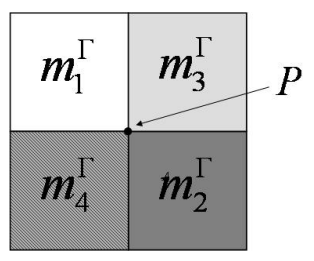

Definition 21 (conflict point). Let $\Theta=\left\{v_{i} \theta_{i j} v_{j}\right\}_{i, j=1}^{n}$ be a basic RCC8 network and $\Gamma=\left\{v_{i}\right.$ $\left.\gamma_{i j} v_{j}\right\}_{i, j=1}^{n}$ a basic RA network. Suppose $\mathfrak{m}^{\Gamma}$ is the canonical solution of $\Gamma$. A point $Q$ is called a conflict point of $m_{i}^{\Gamma}$ if there exists $j$ such that $m_{i}^{\Gamma} \cap m_{j}^{\Gamma}=\{Q\}$ and $\theta_{i j}=\{\mathbf{E C}\}$. We write $C_{i}$ for the set of all conflict points of $m_{i}^{\Gamma}$.

Clearly, each conflict point of $m_{i}^{\Gamma}$ is also a corner point of $m_{i}^{\Gamma}$. This implies that $m_{i}^{\Gamma}$ and $m_{j}^{\Gamma}$ may have at most one common conflict point. Moreover, suppose $\mathfrak{m}=\left(m_{i}\right)_{i=1}^{n}$ is a solution of $\Theta \uplus \Gamma$ such that $\mathcal{M}\left(m_{i}\right)=m_{i}^{\Gamma}$ for all $1 \leq i \leq n$. Then each conflict point of $m_{i}^{\Gamma}$ is contained in $m_{i}$. This means $C_{i} \subset m_{i}$. As a consequence, we have

$$
C_{i} \cap C_{j} \neq \varnothing \Rightarrow \theta_{i j} \neq\{\mathbf{D C}\} \quad(1 \leq i, j \leq n)
$$

The following theorem shows that this is also sufficient.

Theorem 22. Let $\Theta=\left\{v_{i} \theta_{i j} v_{j}\right\}_{i, j=1}^{n}$ be a basic RCC8 network and $\Gamma=\left\{v_{i} \gamma_{i j} v_{j}\right\}_{i, j=1}^{n}$ a basic $R A$ network. Suppose $\Theta \uplus \Gamma$ is bipath-consistent. Then $\Theta \uplus \Gamma$ is satisfiable iff (15) holds.

Proof. The necessity part is clear. We defer the proof of the sufficiency part to Appendix B.

As a corollary, we have $\mathbf{J S P}\left(\mathcal{B}_{\text {rcc } 8}, \mathcal{B}_{\text {rec }}\right)$ is in P.

Corollary 23. For a basic RCC8 network $\Theta$ and a basic RA network $\Gamma$, the consistency of $\Theta \uplus \Gamma$ can be decided in cubic time.

Proof. Bipath-consistency of $\Theta \uplus \Gamma$ can be checked in cubic time. We can construct the unique canonical rectangle solution of $\Gamma$ in quadratic time. The conflict point set $C_{i}$ can also be computed in quadratic time. That is, the condition of Theorem 22 can be checked in cubic time.

\subsection{Large Tractable Subsets}

Recall that RCC8 has three maximal tractable subclasses $\widehat{\mathcal{H}}_{8}, \mathcal{C}_{8}$, and $\mathcal{Q}_{8}$, and IA has one maximal tractable subclass $\mathcal{H}$, all containing the basic relations. In this subsection, we aim to extend the above result to maximal tractable subsets $\widehat{\mathcal{H}}_{8}, \mathcal{C}_{8}$ of RCC8, and the large tractable subset $\mathcal{H} \times \mathcal{H}$ of RA.

To this end, we need to extend the notion of conflict points from basic networks to arbitrary networks. Recall that 0 -meet relations and corner relations are basic RA relations defined in Definition 6.

Definition 24 (common conflict point). Let $\Theta=\left\{v_{i} T_{i j} v_{j}\right\}_{i, j=1}^{n}$ be an RCC8 network and $\Delta=$ $\left\{v_{i} D_{i j} v_{j}\right\}_{i, j=1}^{n}$ an RA network. We say two variables $v_{i}, v_{j}$ have the CCP (common conflict point) relation, written $\operatorname{CCP}\left(v_{i}, v_{j}\right)$, if $D_{i j}$ is a 0 -meet (basic) relation and $T_{i j}=\{\mathbf{E C}\}$, or

- $D_{i j}$ is a (possibly disjunctive) corner relation; and 
- there exist $i^{\prime}, j^{\prime}$ such that $D_{i i^{\prime}}$ and $D_{j j^{\prime}}$ are 0 -meet (basic) relations, $D_{i j^{\prime}}, D_{i^{\prime} j}$ and $D_{i^{\prime} j^{\prime}}$ are (possibly disjunctive) corner relations, and $T_{i i^{\prime}}=T_{j j^{\prime}}=\{\mathbf{E C}\}$.

If $\operatorname{CCP}\left(v_{i}, v_{j}\right)$ and $v_{i}^{\prime}, v_{j}^{\prime}$ are variables that satisfy the above conditions, we also write $\operatorname{CCP}(i, j:$ $\left.i^{\prime}, j^{\prime}\right)$ to stress the roles of $v_{i^{\prime}}$ and $v_{j^{\prime}}$.

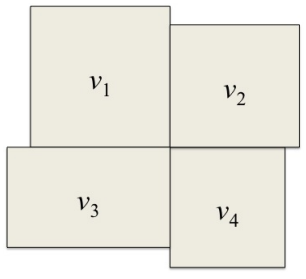

(i)

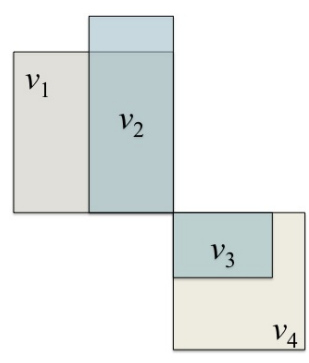

(ii)

Figure 5: Two joint constraint networks in $\mathbf{J S P}(R C C 8, R A)$, where in both (i) and (ii) $D_{i j}$ is the basic RA relation between $v_{i}$ and $v_{j}$ as illustrated in the picture, $T_{14}=T_{23}=\{\mathbf{E C}\}$ and all unspecified RCC8 constraints are non-basic RCC8 relation $\{\mathbf{D C}, \mathbf{E C}, \mathbf{P O}\}$. In both (i) and (ii) we have $\operatorname{CCP}(1,2), \operatorname{CCP}(1,3), \operatorname{CCP}(1,4), \operatorname{CCP}(2,3), \operatorname{CCP}(2,4), \operatorname{CCP}(3,4)$.

Examples are shown in Figure 5. Note that if $\Theta$ and $\Delta$ are all basic networks, then $v_{i}$ and $v_{j}$ have the CCP relation iff $C_{i} \cap C_{j}$ is nonempty, i.e. $v_{i}$ and $v_{j}$ have a common conflict point.

Definition 25. Let $\Theta=\left\{v_{i} T_{i j} v_{j}\right\}_{i, j=1}^{n}$ be an RCC8 network and $\Delta=\left\{v_{i} D_{i j} v_{j}\right\}_{i, j=1}^{n}$ an RA network. We say $\Theta \uplus \Delta$ is CCP-consistent if

$$
\operatorname{CCP}\left(v_{i}, v_{j}\right) \Rightarrow \mathbf{D C} \notin T_{i j}
$$

holds for any $i \neq j$. We say a joint network $\Theta \uplus \Delta$ is $B C$-consistent if it is bipath-consistent and CCP-consistent.

In general, if $v_{i}$ and $v_{j}$ have the CCP relation, then (in any realisation) $v_{i}$ and $v_{j}$ share at least one corner point (of their MBRs) in common. Therefore, in the weak RCC8 algebra, they cannot be disconnected, and neither can be contained in another as a non-tangential proper part. Note that the latter statement also follows from the bi-closedness of $\Theta \uplus \Delta$.

Similar to the bipath-consistency algorithm (Gerevini \& Renz, 2002), we devise an algorithm (Algorithm 1) for enforcing BC-consistency. The following theorem shows that this algorithm is sound.

Theorem 26. Suppose $\Theta \uplus \Delta$ is a joint network of RCC8 and RA constraints, where $\Theta=\left\{v_{i} T_{i j}\right.$ $\left.v_{j}\right\}_{i, j=1}^{n}$ and $\Delta=\left\{v_{i} D_{i j} v_{j}\right\}_{i, j=1}^{n}$. Then in $O\left(n^{4}\right)$ time, the algorithm BC-CONSISTENCY either finds an inconsistency or transforms $\Theta \uplus \Delta$ into an equivalent joint network $\Theta^{\prime} \uplus \Delta^{\prime}$ which is $B C$-consistent. 


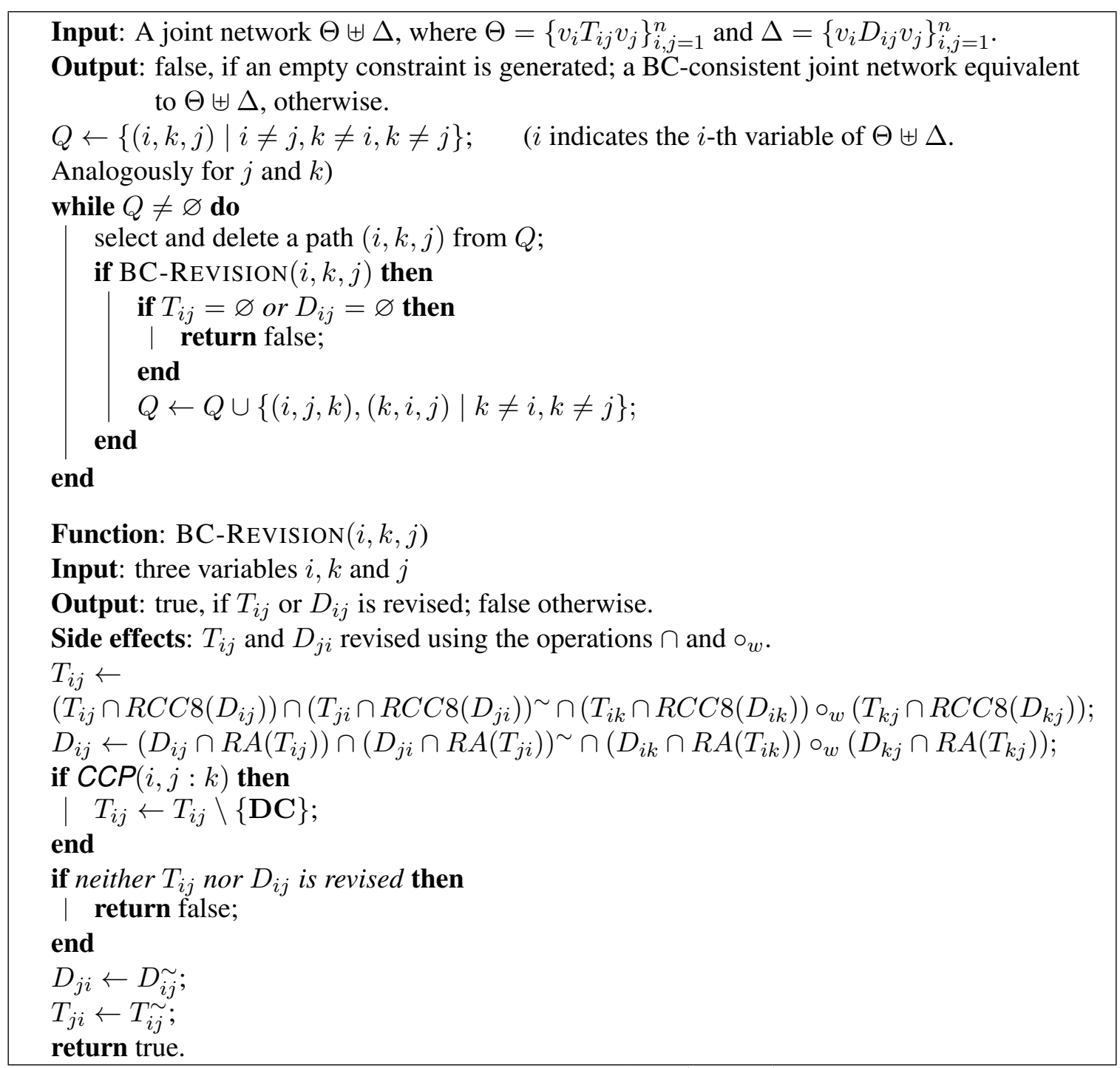

Algorithm 1: BC-CONSISTENCY, where we write $\operatorname{CCP}(i, j: k)$ to represent the situation where there exists another variable $v_{l}$ such that $v_{l}$ and $v_{k}$ together are evidence of $\operatorname{CCP}(i, j)$. 
Proof. This is because, if we iteratively use the following updating rules then either an empty constraint occurs or the network becomes stable.

$$
\begin{aligned}
& T_{i j} \leftarrow\left(T_{i j} \cap \operatorname{RCC} 8\left(D_{i j}\right)\right) \cap\left(T_{j i} \cap \operatorname{RCC} 8\left(D_{j i}\right)\right)^{\sim} \\
& \cap\left(T_{i k} \cap R C C 8\left(D_{i k}\right)\right) \circ_{w}\left(T_{k j} \cap \operatorname{RCC}\left(D_{k j}\right)\right) \\
& D_{i j} \leftarrow\left(D_{i j} \cap R A\left(T_{i j}\right)\right) \cap\left(D_{j i} \cap R A\left(T_{j i}\right)\right)^{\sim} \cap\left(D_{i k} \cap R A\left(T_{i k}\right)\right) \circ_{w}\left(D_{k j} \cap R A\left(T_{k j}\right)\right) \\
& T_{i j} \leftarrow T_{i j} \backslash\{\mathbf{D C}\} \text { if } \operatorname{CCP}(i, j: k),
\end{aligned}
$$

where $i, j, k$ represent the variables $v_{i}, v_{j}$ and $v_{k}$ and $\operatorname{CCP}(i, j: k)$ represents the situation where there exists another variable $v_{l}$ such that $v_{l}$ and $v_{k}$ together are evidence of $\operatorname{CCP}(i, j)$. For each triple, $\operatorname{CCP}(i, j: k)$ can be determined in $O(n)$ time and the subroutine $\operatorname{BC-REVISION}(i, k, j)$ can be carried out in $O(n)$ time. Since each $T_{i j}$ is a set of basic RCC8 relations and each $D_{i j}$ is a set of basic RA relations, $\left(T_{i j}, D_{i j}\right)$ can be revised for a constant number of times. Therefore the number of the loops remains cubic, and BC-CONSISTENCY will terminate in $O\left(n^{4}\right)$ time.

The algorithm is in general not complete. The following lemma will be useful to prove the main result (Theorem 28), which will guarantee the completeness of the algorithm for RCC8 networks over $\widehat{\mathcal{H}}_{8}$ and RA networks over $\mathcal{H} \times \mathcal{H}$.

Lemma 27. Let $\Theta=\left\{v_{i} T_{i j} v_{j}\right\}_{i, j=1}^{n}$ be an RCC8 network and $\Delta=\left\{v_{i} D_{i j} v_{j}\right\}_{i, j=1}^{n}$ an RA network. Suppose $\Theta$ is over $\widehat{\mathcal{H}}_{8}$ or $\mathcal{Q}_{8}$ and $\Theta \uplus \Delta$ is bipath-consistent. Assume that $\Theta^{*}$ is the canonical consistent scenario of $\Theta$ (cf. Theorem 3), and $\Delta^{*}$ is any consistent scenario of $\Delta$. Then $\Theta^{*} \uplus \Delta^{*}$ is bipath-consistent.

Proof. Because both $\Theta^{*}$ and $\Delta^{*}$ are path-consistent basic networks, we need only show that $\Theta^{*} \uplus \Delta^{*}$ is bi-closed, i.e. $\delta_{i j}^{*} \in R A\left(\theta_{i j}^{*}\right)$ and $\theta_{i j}^{*} \in R C C 8\left(\delta_{i j}^{*}\right)$ for any $i \neq j$. Since $\theta_{i j}^{*}$ and $\delta_{i j}^{*}$ are both basic relations, this is equivalent to showing that $\theta_{i j}^{*} \cap \delta_{i j}^{*}$ is nonempty for any $i \neq j$. By (13) and (14) it is straightforward to show that $R A\left(T_{i j}\right)=R A\left(\theta_{i j}^{*}\right)$. Therefore $\delta_{i j}^{*} \subseteq D_{i j} \subseteq R A\left(T_{i j}\right)=R A\left(\theta_{i j}^{*}\right)$, i.e. $\delta_{i j}^{*} \cap \theta_{i j}^{*}$ is nonempty.

We note that this result does not apply to $\mathcal{C}_{8}$. For example, let $T=\{\mathbf{N T P P}, \mathbf{E Q}\}, D=$ $\{\mathrm{d} \otimes \mathrm{d}$, eq $\otimes \mathrm{eq}\}$. The RCC8 relation NTPP is inconsistent with the RA relation eq $\otimes$ eq.

Theorem 28. Let $\Theta=\left\{v_{i} T_{i j} v_{j}\right\}_{i, j=1}^{n}$ be an RCC8 network and $\Delta=\left\{v_{i} D_{i j} v_{j}\right\}_{i, j=1}^{n}$ an RA network. Suppose $\Theta$ is over $\widehat{\mathcal{H}}_{8}$, and $\Delta$ is over $\mathcal{H} \times \mathcal{H}$. Then $\Theta \uplus \Delta$ is consistent if it is BC-consistent.

Proof. Recall that each RA network over $\mathcal{H} \times \mathcal{H}$ is in essence a pair of IA networks over $\mathcal{H}$. By Lemma 8 we know $\Delta$ has a consistent scenario $\Delta^{*}$ such that (i) $\delta_{i j}^{*}$ is a 0 -meet relation iff $D_{i j}$ is; and (ii) $\delta_{i j}^{*}$ is a corner relation iff $D_{i j}$ consists of corner relations. Let $\Theta^{*}$ be the canonical consistent scenario of $\Theta$. We show $\Theta^{*} \uplus \Delta^{*}$ is consistent.

By Lemma 27 we know $\Theta^{*} \uplus \Delta^{*}$ is bipath-consistent. We next show it satisfies (15), which is equivalent to (16) when only basic constraints are concerned. To this end, we show that $\operatorname{CCP}(i, j$ : $\left.i^{\prime}, j^{\prime}\right)$ holds in $\Theta^{*} \uplus \Delta^{*}$ only if it holds in $\Theta \uplus \Delta$. By the choice of $\Delta^{*}$ and $\Theta^{*}$, we know $T_{i i^{\prime}}=$ $T_{j j^{\prime}}=\{\mathbf{E C}\}$ and all the RA relations are either 0-meet relations or consist of corner relations. Therefore, $\operatorname{CCP}\left(i, j: i^{\prime}, j^{\prime}\right)$ also holds in $\Theta \uplus \Delta$. Because $\Theta \uplus \Delta$ is BC-consistent, we know DC is not in $\theta_{i j}$. This implies $\theta_{i j}^{*} \neq\{\mathbf{D C}\}$ and $\Theta^{*} \uplus \Delta^{*}$ satisfies (15). By Theorem 22, we know $\Theta^{*} \uplus \Delta^{*}$, hence $\Theta \uplus \Delta$, is consistent. 
As a consequence, we know the joint consistency problem over $\widehat{\mathcal{H}}_{8}$ or $\mathcal{Q}_{8}$ and $\mathcal{H} \times \mathcal{H}$ can be solved in polynomial time.

Theorem 29. The joint satisfaction problems $\boldsymbol{J S P}\left(\widehat{\mathcal{H}}_{8}, \mathcal{H} \times \mathcal{H}\right)$ and $\boldsymbol{J S P}\left(\mathcal{Q}_{8}, \mathcal{H} \times \mathcal{H}\right)$ are in $P$.

Proof. Suppose $\Theta \uplus \Delta$ is a joint network such that $\Theta$ is over $\widehat{\mathcal{H}}_{8}$ or $\mathcal{Q}_{8}$, and $\Delta$ is over $\mathcal{H} \times \mathcal{H}$. We first apply the algorithm BC-CONSISTENCY to $\Theta \uplus \Delta$. If an empty relation occurs during the process, then $\Theta \uplus \Delta$ is inconsistent. Otherwise, suppose $\Theta^{\prime} \uplus \Delta^{\prime}$ is the BC-consistent joint network equivalent to $\Theta \uplus \Delta$. We assert that $\Theta^{\prime}$ is still over $\widehat{\mathcal{H}}_{8}$ or $\mathcal{Q}_{8}$ and $\Delta^{\prime}$ is over $\mathcal{H} \times \mathcal{H}$. We note that, for any RCC8 relation $T$ in $\widehat{\mathcal{H}}_{8}$ (or $\mathcal{Q}_{8}$ ), and any RA relation $D$ in $\mathcal{H} \times \mathcal{H}$, we have by Lemma 31

- $R C C 8(D)$ is a relation in both $\widehat{\mathcal{H}}_{8}$ and $\mathcal{Q}_{8}$;

- $R A(T)$ is a relation in $\mathcal{H} \times \mathcal{H}$;

- $T \backslash\{\mathbf{D C}\}=T \cap\left\{\mathbf{E C}, \mathbf{P O}, \mathbf{T P P}, \mathbf{N T P P}, \mathbf{T P P} \sim, \mathbf{N T P P}^{\sim}, \mathbf{E Q}\right\}$ is in $\widehat{\mathcal{H}}_{8}\left(\right.$ or $\left.\mathcal{Q}_{8}\right)$.

Because BC-CONSISTENCY only uses the rules (17)-(19) to update relations, each RCC8 relation in $\Theta^{\prime}$ remains in $\widehat{\mathcal{H}}_{8}$ (or $\mathcal{Q}_{8}$ ), and each RA relation in $\Delta^{\prime}$ remains in $\mathcal{H} \times \mathcal{H}$. The consistency $\Theta^{\prime} \uplus \Delta^{\prime}$ then follows from Theorem 28 .

The property in the proof of the above theorem does not hold for $\mathcal{C}_{8}$. It remains open if $\mathbf{J S P}\left(\mathcal{C}_{8}, \mathcal{H} \times \mathcal{H}\right)$ is tractable (though this is not very important for practical purposes since either $\widehat{\mathcal{H}}_{8}$ or $\mathcal{Q}_{8}$ can be used to backtrack over to find a solution if required).

\section{Combination of RCC8' and RA Networks}

In this section, we represent topological information as $\mathrm{RCC} 8$ ' relations and directional information as RA relations. In the previous section we have shown that, for certain tractable subclasses of RCC8 and RA, the JSP can be determined in polynomial time, but we also show that bipath-consistency is incomplete for these subclasses. The reason lies in that two regions that are constrained by DC may have a common conflict point. For RCC $8^{\prime}$, this situation does not exist anymore because two disjoint regions may still have a 0 -dimensional intersection. This section will show that, for RCC $8^{\prime}$, bipath-consistency alone is sufficient to show the consistency of a joint network $\Theta \uplus \Delta$ for $\Theta$ over $\mathcal{H}_{8}$ or $\mathcal{C}_{8}$ and $\Delta$ over $\mathcal{H} \times \mathcal{H}$.

As in the case of weak RCC8, we first consider the interaction between RCC $8^{\prime}$ and RA relations, then consider the consistency of joint basic networks, and, lastly, consider the general case.

Similar to Definition 19, we have the following definition.

Definition 30. Let $T$ be an RCC ${ }^{\prime}$ relation and $D$ an RA relation. The RA relation induced by $T$ and the $\mathrm{RCC} 8^{\prime}$ relation induced by $D$ are defined as

$$
\begin{aligned}
R A(T) & =\{\delta: \delta \text { is a basic RA relation and } \delta \cap T \neq \varnothing\} \\
R C C 8^{\prime}(D) & =\left\{\delta: \theta \text { is a basic RCC } 8^{\prime} \text { relation and } \theta \cap D \neq \varnothing\right\} .
\end{aligned}
$$

It is easy to see that $R A(T)=\bigcup\{R A(\{\theta\}): \theta \in T\}$ and

$$
\begin{aligned}
R A(\{\mathbf{D C}\}) \supset R A(\{\mathbf{E C}\}) \supset R A(\{\mathbf{P O}\}) \supset R A(\{\mathbf{T P P}\})=R A(\{\mathbf{N T P P}\}) \supset R A(\{\mathbf{E Q}\}) . \\
R A(\{\mathbf{P O}\}) \supset R A\left(\left\{\mathbf{T} \mathbf{P} \mathbf{P}^{\sim}\right\}\right)=R A\left(\left\{\mathbf{N T P} \mathbf{P}^{\sim}\right\}\right) \supset R A(\{\mathbf{E Q}\}) .
\end{aligned}
$$


Note that we have $R A(\{\mathbf{T P P}\})=R A(\{\mathbf{N T P P}\})=\{\mathbf{s}, \mathrm{d}, \mathrm{f}$, eq $\} \otimes\{\mathbf{s}, \mathrm{d}, \mathrm{f}$, eq $\}$. This is because in $\mathrm{RCC}^{\prime}$ a non-tangential proper part of a region $a$ may have the same MBR as $a$. For example, each star region in Figure 6 is a non-tangential proper part of its MBR in RCC ${ }^{\prime}$.

Lemma 31. Let $T$ be an $R C C 8^{\prime}$ relation and $D$ an $R A$ relation. Then $R C C 8^{\prime}(D)$ is a relation in the intersection of $\widehat{\mathcal{H}}_{8}, \mathcal{Q}_{8}$, and $\mathcal{C}_{8}$; and $R A(T)$ is a relation in $\mathcal{H} \times \mathcal{H}$ if $T$ is a relation in $\widehat{\mathcal{H}}_{8}$ or $\mathcal{Q}_{8}$ or $\mathcal{C}_{8}$.

In particular, unlike the case for weak RCC8, we have $R A(\{\mathbf{N T P P}, \mathbf{E Q}\})=\{\mathbf{s}, \mathrm{d}, \mathrm{f}$, eq $\} \otimes$ $\{\mathrm{s}, \mathrm{d}, \mathrm{f}, \mathrm{eq}\}$ is a relation in $\mathcal{H} \times \mathcal{H}$.

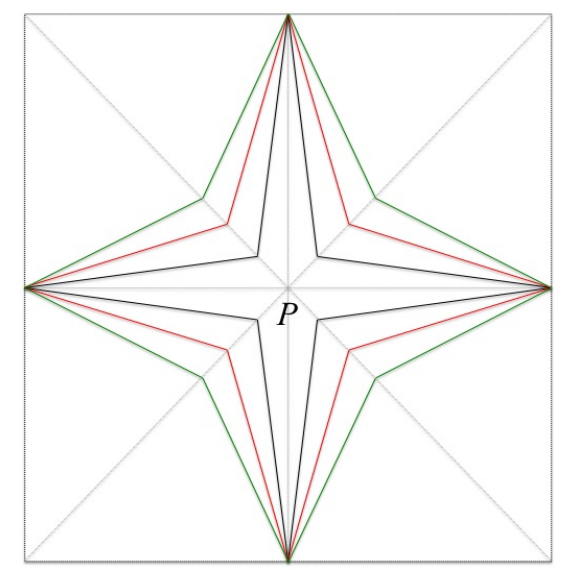

Figure 6: Basic regions of a control point $P$ in the combination of $\mathrm{RCC} 8^{\prime}$ and RA.

Theorem 32. Suppose $\Theta$ is a basic RCC $8^{\prime}$ network and $\Delta$ is a basic RA network. Then $\Theta \uplus \Delta$ is consistent if it is bipath-consistent.

Proof. The proof follows the same pattern as for the combination of weak RCC8 and RA (Theorem 22), but we need to replace the basic regions around a control point $P$ with the star regions shown in Figure 6, where we only show three regions $b, r, g$ around $P$, and $b \mathbf{N T P P} r$ and $r \mathbf{N T P P} g$.

We have the following result for $\mathrm{RCC} 8^{\prime}$ and RA.

Theorem 33. Suppose $\Theta$ is a network over $\widehat{\mathcal{H}}_{8}$ or $\mathcal{Q}_{8}$ in $R C C 8^{\prime}, \Delta$ is an RA network. Then $\Theta \uplus \Delta$ is consistent if $\Theta \uplus \Delta$ is bi-closed, $\Theta$ is path-consistent, and $\Delta$ is consistent.

Proof. Assume that $\Theta^{*}$ is the canonical consistent scenario of $\Theta$, and $\Delta^{*}$ is any consistent scenario of $\Delta$. Then, completely similar to Lemma 27, we can show that $R A\left(\theta_{i j}^{*}\right)=R A\left(\theta_{i j}\right)$ and hence the bi-closeness of $\Theta^{*} \uplus \Delta^{*}$. Because $\Theta^{*}$ and $\Delta^{*}$ are consistent, we know $\Theta^{*} \uplus \Delta^{*}$ is bipath-consistent, hence consistent by Theorem 32 .

The above result shows that the consistency of a joint network in $\operatorname{JSP}\left(\widehat{\mathcal{H}}_{8}, R A\right)$ can be polynomially reduced to determining the consistency of an RCC8 network over $\widehat{\mathcal{H}}_{8}$ and an RA network. In this sense, $\mathbf{J S P}\left(\widehat{\mathcal{H}}_{8}, R A\right)$ is a separable problem. In particular, we have 
Theorem 34. If RCC8 relations are interpreted by using strong connectedness, then the joint satisfaction problems $\boldsymbol{J} \boldsymbol{S P}\left(\widehat{\mathcal{H}}_{8}, \mathcal{H} \times \mathcal{H}\right)$ and $\boldsymbol{J S P}\left(\mathcal{Q}_{8}, \mathcal{H} \times \mathcal{H}\right)$ are in $P$.

Again, it remains open whether the above result holds for networks over $\mathcal{C}_{8}$ in $\mathrm{RCC} 8^{\prime}$, even though in this case $R A(\{\mathbf{N T P P}, \mathbf{E Q}\})=R A(\mathbf{T P P})$ is a relation in $\mathcal{H} \times \mathcal{H}$.

In the following section, we consider the combination of RCC8 and CDC constraints.

\section{Combination of RCC8 and CDC Constraints}

Although basic RCC8 networks and basic CDC networks can be solved in cubic time independently, the interaction between RCC 8 and CDC constraints makes the joint satisfaction problem hard to solve. In this section, we first show that the joint satisfaction problem is in NP by designing a polynomial non-deterministic algorithm and then show it is NP-hard even for basic constraints. This shows that $\mathbf{J S P}\left(\mathcal{B}_{r c c 8}, \mathcal{B}_{c d c}\right)$ is NP-complete. We then consider three variants of $\operatorname{JSP}\left(\mathcal{B}_{r c c 8}, \mathcal{B}_{c d c}\right)$ obtained by replacing RCC8 with RCC $8^{\prime}$ and/or CDC with OIM. Write $\mathcal{B}_{\text {oim }}$ for the set of basic OIM relations. We show $\mathbf{J S P}\left(\mathcal{B}_{r c c 8}, \mathcal{B}_{o i m}\right)$ and $\mathbf{J S P}\left(\mathcal{B}_{r c c 8^{\prime}}, \mathcal{B}_{c d c}\right)$ are NP-complete, but $\mathbf{J S P}\left(\mathcal{B}_{r c c 8^{\prime}}, \mathcal{B}_{\text {oim }}\right)$ is in $\mathrm{P}$.

\subsection{Algorithms}

Let $\Theta$ be an instance of a joint basic RCC8 or RCC $8^{\prime}$ network and $\Delta$ a basic CDC or OIM network over the same set of variables. We provide in this subsection algorithms for determining the consistency of $\Theta \uplus \Delta$. Our key idea is first showing that $\Theta \uplus \Delta$ is consistent iff $\Delta$ has a regular solution that is RA consistent with $\Theta$ (see below) and then giving algorithms for determining whether $\Delta$ has such a regular solution.

Suppose $\mathfrak{m}=\left(m_{i}\right)_{i=1}^{n}$ is a solution of $\Delta$. Recall that we say $\mathfrak{m}=\left(m_{i}\right)_{i=1}^{n}$ is a regular solution if it is a maximal solution and $\left\{\mathcal{M}\left(m_{i}\right)\right\}_{i=1}^{n}$ is a canonical tuple of rectangles (cf. Dfn. 13). Note that each region in a regular solution $\mathfrak{m}$ is the union of a set of cells introduced by the canonical tuple of rectangles.

Definition 35. Let $\Theta=\left\{v_{i} \theta_{i j} v_{j}\right\}_{i, j=1}^{n}$ be a basic RCC8 network and $\Delta=\left\{v_{i} \delta_{i j} v_{j}\right\}_{i, j=1}^{n}$ a basic CDC network. Suppose $\mathfrak{m}=\left(m_{i}\right)_{i=1}^{n}$ is a regular solution of $\Delta$. Write $\Gamma$ for the RA network induced by $\mathfrak{m}$. We say a regular solution $\mathfrak{m}$ of $\Delta$ is $R A$ consistent with $\Theta$ if there exists a solution of $\Theta \uplus \Delta$ which also satisfies $\Gamma$.

The following lemma gives a characterisation of consistent joint basic networks.

Lemma 36. Let $\Theta=\left\{v_{i} \theta_{i j} v_{j}\right\}_{i, j=1}^{n}$ be a basic RCC8 network and $\Delta=\left\{v_{i} \delta_{i j} v_{j}\right\}_{i, j=1}^{n}$ a basic $C D C$ network. Then $\Theta \uplus \Delta$ is consistent iff $\Delta$ has a regular solution that is RA consistent with $\Theta$.

Proof. The sufficiency part is clear by definition. We only prove the necessity part. Suppose $\mathfrak{a}=$ $\left(a_{i}\right)_{i=1}^{n}$ is a solution of $\Theta \uplus \Delta$. Write $\Gamma$ for the RA network induced by $\mathfrak{a}$. Then $\mathfrak{a}$ is also a solution of $\Delta \uplus \Gamma$. Hence there is a unique regular solution of $\Delta$ which also satisfies $\Gamma$. Write $\mathfrak{m}=\left(m_{i}\right)_{i=1}^{n}$ for this regular solution. It is clear that $\mathfrak{m}$ is a regular solution of $\Delta$ that is RA consistent with $\Theta$.

By this lemma, to determine the consistency of $\Theta \uplus \Delta$, we need only determine the existence of regular solutions of $\Delta$ that are RA consistent with $\Theta$. Suppose $\mathfrak{m}$ is a regular solution of $\Delta$. We next give a necessary and sufficient condition for $\mathfrak{m}$ being RA consistent with $\Theta$. 
To this end, we first fix some notation and terminology. For a region $m_{i}$ in $\mathfrak{m}$, we say a corner point $P$ of $m_{i}$ is a potential conflict point (in $\mathfrak{m}$ ) if exactly one of the four cells incident to $P$ is contained in $m_{i}$. For example, the grey region shown in Figure 7 has five potential conflict points $P_{i}(i=1, \ldots, 5)$. Later we will show that these points may introduce conflicts that are hard to resolve when RCC8 constraints are involved. Furthermore, we denote by $G_{i}$ the set of cells contained in $m_{i}, E_{i}$ the set of edges of cells which lie on the boundary of $m_{i}$, and $N_{i}$ the set of potential conflict points of $v_{i}$.

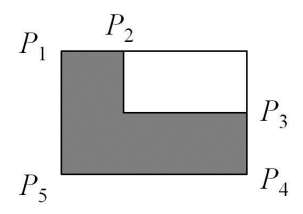

Figure 7: Illustration of potential conflict points.

Lemma 37. Let $\Theta=\left\{v_{i} \theta_{i j} v_{j}\right\}_{i, j=1}^{n}$ be a basic RCC8 network and $\Delta=\left\{v_{i} \delta_{i j} v_{j}\right\}_{i, j=1}^{n}$ a basic $C D C$ network. If a regular solution $\mathfrak{m}=\left(m_{i}\right)_{i=1}^{n}$ of $\Delta$ is $R A$ consistent with $\Theta$, then we have:

$$
\text { if } \theta_{i j}=\mathbf{T P P} \text { or } \mathbf{N T P P} \text { then } G_{i} \subseteq G_{j} \text {. }
$$

Proof. We prove this by contradiction. Assume that $\left(v_{i} \mathbf{T P P} v_{j}\right)$ or $\left(v_{i} \mathbf{N T P} \mathbf{P} \mathbf{P} v_{j}\right)$ is a constraint in $\Theta$ and $G_{i} \nsubseteq G_{j}$.

Suppose $\left(v_{s} \delta v_{t}\right)$ is a constraint in $\Delta$, where $\delta$ is a basic CDC relation represented by the $3 \times 3$ Boolean matrix $\left(d^{p q}\right)_{1 \leq p, q \leq 3}$. Because $\mathfrak{m}$ is a solution of $\Delta$, we have for any $1 \leq p, q \leq 3$ that

$$
d^{p q}=1 \quad \text { iff } \quad m_{s}^{\circ} \cap m_{t}^{p q} \neq \varnothing,
$$

where $m_{t}^{p q}$ denotes one of the nine tiles generated by the MBR of $m_{t}$ (cf. Fig. 3). Since $\mathfrak{m}$ is a regular solution and $G_{s}$ is the set of cells contained in $m_{s}$, this is equivalent to saying that

$$
d^{p q}=1 \quad \text { iff } \quad G_{s} \text { and } m_{t}^{p q} \text { have a common cell. }
$$

Now let $g$ be a cell in $G_{i} \backslash G_{j}$. Because $g$ is not in $G_{j}$, by the construction procedure of regular solutions (see Appendix A), there exists a constraint $\left(v_{j} \delta^{\prime} v_{k}\right) \in \Delta$ with $\delta^{\prime}=\left(d^{\prime u v}\right)$ such that $g$ is a cell contained in $m_{k}^{p q}$ for some $p, q$. By (24) and that $g$ is not in $G_{j}$ we know $d^{\prime p q}=0$ and hence, by (23), $m_{j}^{\circ} \cap m_{k}^{p q}=\varnothing$. Let $\left(v_{i} \delta^{\prime \prime} v_{k}\right)$ be the CDC constraint between $v_{i}$ and $v_{k}$ in $\Delta$ and suppose $\delta^{\prime \prime}=\left(d^{\prime \prime u v}\right)$. Because $g$ is cell in both $G_{i}$ and $m_{k}^{p q}$, we have $d^{\prime \prime p q}=1$ by (24).

Because $\mathfrak{m}$ is RA consistent with $\Theta$, there exists a solution $\mathfrak{a}=\left(a_{i}\right)_{i=1}^{n}$ of $\Theta \uplus \Delta$ such that $\mathcal{M}\left(a_{i}\right)=\mathcal{M}\left(m_{i}\right)$. Since $\left(v_{i} \mathbf{T} \mathbf{P} \mathbf{P} v_{j}\right)$ or $\left(v_{i} \mathbf{N T P} \mathbf{P} v_{j}\right)$ is in $\Theta$, we know $a_{i} \subset a_{j}$. Furthermore, we have $a_{j} \subseteq m_{j}$ as $\mathfrak{m}$ is a maximal solution of $\Delta$. Therefore, $a_{i} \subset m_{j}$. Because $m_{k}^{p q}=a_{k}^{p q}$ and $m_{j}^{\circ} \cap m_{k}^{p q}=\varnothing, a_{i}^{\circ} \cap a_{k}^{p q}$ is empty. This shows that $\left(a_{i}, a_{k}\right)$ is not in $\delta^{\prime \prime}$ since $d^{\prime \prime p q}=1$. A contradiction.

The NTPP constraints may furthermore exclude some edges in $E_{i}$ and nodes in $N_{i}$ from the valuation of $v_{i}$. Suppose $v_{i} \mathbf{N T P P} v_{j}$ is a constraint in $\Theta$ and $\mathfrak{m}=\left(m_{i}\right)_{i=1}^{n}$ is RA consistent with $\Theta$. For any solution $\mathfrak{a}=\left(a_{i}\right)_{i=1}^{n}$ of $\Theta \uplus \Delta$ with $\mathcal{M}\left(a_{i}\right)=\mathcal{M}\left(m_{i}\right)$, by $a_{i} \mathbf{N T P P} a_{j}$ and $a_{j} \subseteq m_{j}$ 
we have $a_{j} \cap \partial m_{j}=\varnothing$. This is to say, $a_{i}$ cannot touch the edges and nodes in $E_{j}$ and $N_{j}$. To characterise this, we define

$$
\begin{aligned}
& \bar{E}_{i} \equiv E_{i} \backslash \bigcup\left\{E_{j}: v_{i} \mathbf{N T P P} v_{j} \in \Theta\right\}, \\
& \bar{N}_{i} \equiv N_{i} \backslash \bigcup\left\{N_{j}: v_{i} \mathbf{N T P P P} v_{j} \in \Theta\right\} .
\end{aligned}
$$

Since every region in $\mathfrak{m}$ can be represented by a Boolean matrix, $G_{i}, \bar{E}_{i}, \bar{N}_{i}$ can be calculated in polynomial time. The following proposition then gives a necessary and sufficient condition for $\mathfrak{m}$ being RA consistent with $\Theta$.

Lemma 38. Let $\Theta=\left\{v_{i} \theta_{i j} v_{j}\right\}_{i, j=1}^{n}$ be a basic RCC8 network and $\Delta=\left\{v_{i} \delta_{i j} v_{j}\right\}_{i, j=1}^{n}$ a basic $C D C$ network. Then a regular solution $\mathfrak{m}=\left(m_{i}\right)_{i=1}^{n}$ of $\Delta$ is $R A$ consistent with $\Theta$ iff

- $v_{i} \mathbf{T P P} v_{j} \in \Theta$ or $v_{i} \mathbf{N T P P} v_{j} \in \Theta$ implies $G_{i} \subseteq G_{j}$, and

- $\mathcal{M}\left(\bigcup \bar{E}_{i}\right)=\mathcal{M}\left(m_{i}\right)$ for any $i$, and

- $v_{i} \mathbf{P O} v_{j} \in \Theta$ implies $G_{i} \cap G_{j} \neq \varnothing$, and

- there exists a resolving function $f$, which is defined as a function from $V$ to $\mathcal{P}\left(\bigcup\left\{\bar{N}_{1}, \cdots, \bar{N}_{n}\right\}\right)$ satisfying (27)-(29).

$$
\begin{aligned}
& f\left(v_{i}\right) \subseteq \bar{N}_{i}, \\
& v_{i} \mathbf{E C} v_{j} \in \Theta \Rightarrow G_{i} \cap G_{j} \neq \varnothing \text { or } \bar{E}_{i} \cap \bar{E}_{j} \neq \varnothing \text { or } f\left(v_{i}\right) \cap f\left(v_{j}\right) \neq \varnothing, \\
& v_{i} \mathbf{D C} v_{j} \in \Theta \Rightarrow f\left(v_{i}\right) \cap f\left(v_{j}\right)=\varnothing .
\end{aligned}
$$

Proof. We begin with the necessity part. Suppose $\mathfrak{m}$ is RA consistent with $\Theta$. Then by definition there exists a solution $\mathfrak{a}=\left(a_{i}\right)_{i=1}^{n}$ of $\Theta \uplus \Delta$ such that $\mathcal{M}\left(a_{i}\right)=\mathcal{M}\left(m_{i}\right)$. The first condition is proven in Lemma 37. For the second condition, because $\mathcal{M}\left(a_{i}\right)=\mathcal{M}\left(m_{i}\right)$, and $a_{i} \subseteq m_{i}$, we know that $a_{i}$ has a nonempty intersection with one unit edge on a cell that lies on the top (bottom, leftmost, or rightmost) edge of $\mathcal{M}\left(m_{i}\right)$. This unit edge is clearly in $E_{i}$. Furthermore, it can be proven that this edge is in $\bar{E}_{i}$, and thus we have $\mathcal{M}\left(\bigcup \bar{E}_{i}\right)=\mathcal{M}\left(m_{i}\right)$. The following two conditions guarantee that the PO, EC constraints can be satisfied while not violating DC constraints. The third condition follows directly from $a_{i} \subseteq \bigcup G_{i}$ and $a_{i} \mathbf{P O} a_{j}$. For the last condition, we define a resolving function $f$ as $f\left(v_{i}\right)=\left\{P \in \bar{N}_{i}: P \in a_{i}\right\}$. It is straightforward to prove that $f$ satisfies (27)-(29).

For the sufficiency part, we construct a solution of $\Theta \uplus \Delta$. The procedure is quite similar to that given for Theorem 22 in Appendix B. For $v_{i}$, we choose a control point from each cell in $G_{i}$ and a control point from each edge in $\bar{E}_{i}$. If $v_{i} \mathbf{P O} v_{j}$, we choose a control point for both of them from a common cell of $G_{i}$ and $G_{j}$. If $v_{i} \mathbf{E C} v_{j}$, we choose a control point for them in a common cell if $G_{i} \cap G_{j} \neq \varnothing$, or from a common edge if $\bar{E}_{i} \cap \bar{E}_{j} \neq \varnothing$, or from $f\left(v_{i}\right) \cap f\left(v_{j}\right)$ by the resolving function $f$. It can then be proven that these control points lead to a solution of $\Theta$. Moreover, the choice of control points ensures that the regions are also a solution of $\Delta$.

Since the conditions in Lemma 38 can be verified by a nondeterministic polynomial algorithm, we have the following theorem.

Theorem 39. $\boldsymbol{J S P}\left(\mathcal{B}_{r c c 8}, \mathcal{B}_{c d c}\right)$ is in $N P$. 
Proof. Suppose $\Theta \uplus \Delta$ is an instance of $\mathbf{J S P}\left(\mathcal{B}_{r c c 8}, \mathcal{B}_{c d c}\right)$. We devise a nondeterministic polynomial algorithm as follows. We first guess a basic RA network $\Gamma$ that is consistent with the basic CDC network $\Delta$, and compute the regular solution $\mathfrak{m}$ of $\Delta$ that satisfies $\Gamma$ in cubic time, and further guess a resolving function $f$ that satisfies the conditions (27)-(29), and check whether $\mathfrak{m}$ is consistent with the basic RCC8 network $\Theta$ by Lemma 38 via $f$. If this is the case, the algorithm returns true. Otherwise, the algorithm returns false. This shows that $\mathbf{J S P}\left(\mathcal{B}_{r c c 8}, \mathcal{B}_{c d c}\right)$ is in NP.

Since each OIM network has at most one regular solution, by Lemma 38 we have

Corollary 40. $\boldsymbol{J S P}\left(\mathcal{B}_{\text {rcc } 8}, \mathcal{B}_{\text {oim }}\right)$ is in $N P$.

If we interpret topological constraints in RCC ${ }^{\prime}$, then we have the following simplified condition for determining whether a regular solution of $\Delta$ is RA consistent with $\Theta$.

Proposition 41. Suppose that $\Theta$ is a basic RCC8' network, and $\triangle$ is a basic CDC network, both over the same set of variables $V=\left\{v_{1}, \cdots, v_{n}\right\}$. Then a regular solution of $\Delta$ is $R A$ consistent with $\Theta$ iff

- $v_{i} \mathbf{T P P} v_{j} \in \Theta$ or $v_{i} \mathbf{N T P P} v_{j} \in \Theta$ implies $G_{i} \subseteq G_{j}$, and

- $v_{i} \mathbf{P O} v_{j} \in \Theta$ implies $G_{i} \cap G_{j} \neq \varnothing$, and

- $v_{i} \mathbf{E C} v_{j} \in \Theta$ implies that either $G_{i} \cap G_{j}$ or $\bar{E}_{i} \cap \bar{E}_{j}$ is nonempty.

Moreover, the above conditions can be checked in polynomial time.

Proof. The proof is similar to that for Lemma 38. The resolving function is now irrelevant, because in $\mathrm{RCC}^{\prime}$ conflict points are no longer evidence for $\mathbf{E C}$ relations, where a point $P$ (a set $X$, respectively) is regarded as evidence of a relation $(a \mathbf{E C} b)$ if $P \in a \cap b(X \subseteq a \cap b)$, respectively. Note that we do not require $\mathcal{M}\left(\bigcup \bar{E}_{i}\right)=\mathcal{M}\left(m_{i}\right)$, as in $\mathrm{RCC}^{\prime}$ it is possible that $a \mathbf{N T P P} b$ and $\mathcal{M}(a)=\mathcal{M}(b)$, see Figure 6 for illustration.

This directly leads to the following two results.

Theorem 42. $\boldsymbol{J S P}\left(\mathcal{B}_{r c c 8^{\prime}}, \mathcal{B}_{c d c}\right)$ is in $N P$.

Proof. The proof is similar to that for Theorem 39. Suppose $\Theta \uplus \Delta$ is an instance of $\mathbf{J S P}\left(\mathcal{B}_{r c c 8}, \mathcal{B}_{c d c}\right)$. We first guess a basic RA network $\Gamma$ that is consistent with $\Delta$ and construct a regular solution $\mathfrak{m}$ of $\Delta$ that satisfies $\Gamma$ and then check whether $\mathfrak{m}$ is RA consistent with $\Theta$ by Proposition 41 .

Recall that OIM is in essence the combination of CDC and RA, and a basic OIM network is consistent iff the two component CDC and RA networks are consistent (see Proposition 17). In the case when $\mathrm{RCC} 8^{\prime}$ is combined with OIM, we have the following tractability result.

Theorem 43. $\boldsymbol{J S P}\left(\mathcal{B}_{r c c 8^{\prime}}, \mathcal{B}_{\text {oim }}\right)$ is in $P$.

Proof. The algorithm for $\mathbf{J S P}\left(\mathcal{B}_{r c c 8^{\prime}}, \mathcal{B}_{\text {oim }}\right)$ contains three steps. Suppose $\Theta \uplus \Delta$ is an instance with $n$ variables. The first step is to decide whether $\Delta$ and $\Theta$ are independently consistent. If not so, then return false; otherwise, construct the unique regular solution $\mathfrak{m}$ of $\Delta$. This can be achieved in $O\left(n^{3}\right)$ time. We then calculate $G_{i}$ and $\bar{N}_{i}$, which can be done in $O\left(n^{4}\right)$ time. The third step is to decide whether $\mathfrak{m}$ is RA consistent with $\Theta$ according to Proposition 41, which can be done in $O\left(n^{4}\right)$ time. Therefore, the consistency of $\Theta \uplus \Delta$ can be determined in $O\left(n^{4}\right)$ time and, hence, $\operatorname{JSP}\left(\mathcal{B}_{r c c 8^{\prime}}, \mathcal{B}_{\text {oim }}\right)$ is in P. 
In the next subsection, we show that $\mathbf{J S P}\left(\mathcal{B}_{r c c 8}, \mathcal{B}_{c d c}\right), \mathbf{J S P}\left(\mathcal{B}_{r c c 8^{\prime}}, \mathcal{B}_{c d c}\right)$, and $\mathbf{J S P}\left(\mathcal{B}_{r c c 8}, \mathcal{B}_{\text {oim }}\right)$ are all NP-hard.

\subsection{NP-Hardness Results}

Recall that in the proof of Theorem 39, we guess twice when determining the consistency of an instance $\Theta \uplus \Delta$ of $\mathbf{J S P}\left(\mathcal{B}_{r c c 8}, \mathcal{B}_{c d c}\right)$, once for a basic RA network that is consistent with $\Delta$, and once for a resolving function $f$ that satisfies (27)-(29) (see Proposition 38). In this subsection we devise two polynomial reductions from known NP-hard problems to $\mathbf{J S P}\left(\mathcal{B}_{r c c 8}, \mathcal{B}_{c d c}\right)$ by exploiting these two facts.

Theorem 44. $\boldsymbol{J S P}\left(\mathcal{B}_{r c c 8}, \mathcal{B}_{c d c}\right)$ is NP-hard.

Proof. The first reduction is from 3-SAT to $\mathbf{J S P}\left(\mathcal{B}_{r c c 8}, \mathcal{B}_{c d c}\right)$. Because it is quite complicated, we defer the construction to Appendix C. Here we only explain why this problem is NP-hard.

For each 3-SAT instance $\varphi$, we construct an instance $\Theta_{\varphi} \uplus \Delta_{\varphi}$ of $\mathbf{J S P}\left(\mathcal{B}_{r c c 8}, \mathcal{B}_{c d c}\right)$ such that each RCC8 constraint is either a DC or an EC constraint. Furthermore, we can show that $\Delta_{\varphi}$ has a unique regular solution that is RA consistent with $\Theta_{\varphi}$ if $\varphi$ is consistent.

The intractability is caused by the potential conflict points in the regular solution, which, together with the EC and DC constraints, may introduce conflicts that are hard to resolve. By Lemma 38, to satisfy an EC constraint $v_{i} \mathbf{E C} v_{j}$, we need to check whether $m_{i}$ and $m_{j}$ share a cell, or else an edge, or else a corner point. In the last case, it can be proven without much difficulty that points shared by $m_{i}$ and $m_{j}$ are exactly those points in $\bar{N}_{i} \cap \bar{N}_{j}$. Therefore, if $m_{i}$ and $m_{j}$ share no cell or edge, then the evidence point for the constraint $v_{i} \mathbf{E} \mathbf{C} v_{j}$ can only be chosen from $\bar{N}_{i} \cap \bar{N}_{j}$. It turns out that choosing such evidence points for all the EC constraints while not violating the DC constraints in $\Theta$ is NP-hard.

The second reduction is from Graph 3-colouring problem to $\mathbf{J S P}\left(\mathcal{B}_{r c c 8}, \mathcal{B}_{c d c}\right)$. We defer the construction to Appendix D. For each graph $G$, we construct an instance $\Theta_{G} \uplus \Delta_{G}$ in $\mathbf{J S P}\left(\mathcal{B}_{r c c 8}, \mathcal{B}_{c d c}\right)$. This reduction differs from the first one in that it does not exploit the intractability of finding a resolving function. In fact, when $v_{i} \mathbf{E C} v_{j}$ is a constraint, then in each regular solution $\mathfrak{m}$ of $\Delta_{G}$, either $m_{i}$ and $m_{j}$ share a cell or an edge, or $m_{i}$ and $m_{j}$ are disjoint (in which case $\mathfrak{m}$ is not RA consistent with $\Theta$ ). That is to say, resolving functions have no effect on the RA consistency of $\mathfrak{m}$. The reduction is based on the fact that $\Delta_{G}$ has exponentially many regular solutions, and there is no general way to test all of them in polynomial time (unless $\mathrm{P}=\mathrm{NP}$ ).

Note that in the first reduction we have shown $\Delta_{\varphi}$ has a unique regular solution that is RA consistent with $\Theta_{\varphi}$ if $\varphi$ is consistent, where $\varphi$ is a 3-SAT instance and $\Theta_{\varphi} \uplus \Delta_{\varphi}$ is the instance of $\operatorname{JSP}\left(\mathcal{B}_{r c c 8}, \mathcal{B}_{c d c}\right)$ defined by the reduction. Write $\Gamma_{\varphi}$ for the basic RA network induced by this particular regular solution of $\Delta_{\varphi}$. It is easy to see that $\Theta_{\varphi} \uplus \Delta_{\varphi}$ is consistent iff $\Theta_{\varphi} \uplus \Delta_{\varphi} \uplus \Gamma_{\varphi}$ is consistent. In other words, the reduction from 3-SAT is also a reduction to $\mathbf{J S P}\left(\mathcal{B}_{\text {rcc } 8}, \mathcal{B}_{\text {oim }}\right)$.

Corollary 45. $\boldsymbol{J S P}\left(\mathcal{B}_{r c c 8}, \mathcal{B}_{\text {oim }}\right)$ is NP-hard.

Similarly, the second reduction is also a reduction to $\mathbf{J S P}\left(\mathcal{B}_{r c c 8^{\prime}}, \mathcal{B}_{c d c}\right)$. This is because the $\mathbf{J S P}\left(\mathcal{B}_{r c c 8}, \mathcal{B}_{c d c}\right)$ instance for each graph $G$ only uses DC and EC constraints, and when two variables are required to be EC, then their MBRs do not 0-meet, but their MBRs may overlap.

Corollary 46. $\boldsymbol{J S P}\left(\mathcal{B}_{r c c 8^{\prime}}, \mathcal{B}_{c d c}\right)$ is $N P$-hard. 
By these NP-hardness results and Theorem 39, Corollary 40, and Theorem 42, we know

Theorem 47. The joint satisfaction problems $\boldsymbol{J S P}\left(\mathcal{B}_{r c c 8}, \mathcal{B}_{c d c}\right), \boldsymbol{J S P}\left(\mathcal{B}_{r c c 8^{\prime}}, \mathcal{B}_{c d c}\right)$, and $\boldsymbol{J S P}\left(\mathcal{B}_{r c c 8}\right.$, $\left.\mathcal{B}_{\text {oim }}\right)$ are all NP-complete.

\section{Conclusion}

In this paper, we have investigated the computational complexity of reasoning with topological relations and cardinal directions between extended spatial objects. We used two different interpretations of the well-known RCC8 algebra for representing topological information, and use the Rectangle Algebra (RA) and the cardinal direction calculus (CDC) to describe directional information. We have shown that the joint satisfaction problems are decidable and remain in NP for all these interpretations of topological and directional information. More importantly, we have shown that the consistency problem is in P when basic (weak or strong) RCC 8 and basic RA constraints are involved, or when topological constraints are basic strong RCC8 constraints and directional constraints are jointly represented by basic RA and CDC constraints.

Some related work has been reported in the work of Sistla and Yu (2000), Li (2007), and Li and Cohn (2012), but only small fragments of RA are used to express directional information. Our results represent a large step towards the applicability of qualitative spatial reasoning techniques for real-world problems. In particular the tractable results are very promising as they enable efficient reasoning about these important calculi. It also means that if efficient reasoning is important for a potential application, developers should aim for representing directional information using RA (or together with $\mathrm{CDC}$ ) instead of $\mathrm{CDC}$ alone and/or representing topological information by using RCC8' instead of RCC8. Our results about combining RCC8 and CDC/OIM are very important from a theoretical point of view as they are the first formal results for this combination. As demonstrated using a concrete application scenario, our results are also of practical significance, as the combined information we consider can be easily extracted in cases where computer vision is used for obtaining spatial information.

\section{Acknowledgments}

We thank the anonymous reviewers for their invaluable comments and detailed suggestions. The first author also thanks the University of Technology Sydney for their funding of a visit to Sydney as an Adjunct Professor. This work was partially supported by Australian Research Council (Grant No.s DP120104159, DP120103758 and FT0991917), the National Natural Science Foundation of China (Grant No. 61228305), and the EU funded projects RACE (FP7-ICT-287752) and STRANDS (FP7-ICT-600623).

\section{Appendix A. Realisation of Basic CDC Networks}

We here describe the cubic algorithm given in the work of Zhang et al., (2008), and Liu et al., (2010). Given a basic CDC network, first, we compute a canonical solution of the induced (possibly nonbasic) RA network. Next, we remove the cells that violate some constraints from each rectangle. Third, we check whether what we have obtained is a valid solution. In the following, we give a detailed description with a running example illustrated in Table 4 and Figure 8. 


\begin{tabular}{c|ccccc}
\hline & $\delta_{i j}$ & $\delta_{j i}$ & $\iota_{i j}^{x} \otimes \iota_{i j}^{y}$ & $\rho_{i j}^{x} \otimes \rho_{i j}^{y}$ \\
\hline$(1,2)$ & {$\left[\begin{array}{lll}0 & 0 & 0 \\
1 & 1 & 0 \\
1 & 0 & 0\end{array}\right]$} & {$\left[\begin{array}{lll}0 & 1 & 1 \\
0 & 1 & 1 \\
0 & 0 & 0\end{array}\right]$} & $\mathbf{0} \otimes \mathbf{o}$ & $\mathbf{0} \otimes \mathbf{o}$ \\
$(1,3)$ & {$\left[\begin{array}{lll}0 & 0 & 0 \\
1 & 0 & 0 \\
1 & 0 & 0\end{array}\right]$} & {$\left[\begin{array}{lll}0 & 0 & 0 \\
0 & 1 & 0 \\
0 & 0 & 0\end{array}\right] \quad\{\mathrm{m}, \mathrm{b}\} \otimes \mathrm{fi}$} & $\mathbf{b} \otimes \mathrm{fi}$ \\
$(2,3)$ & {$\left[\begin{array}{lll}1 & 1 & 0 \\
1 & 0 & 0 \\
0 & 0 & 0\end{array}\right] \quad\left[\begin{array}{lll}0 & 0 & 0 \\
0 & 0 & 1 \\
0 & 1 & 1\end{array}\right]$} & $\mathbf{0} \otimes$ oi & $\mathbf{0} \otimes$ oi \\
\hline
\end{tabular}

Table 4: Example of solving a basic CDC network.

Step 1. Compute the induced RA network $\Gamma^{0}$ of $\Delta$.

Step 2. Refine $\Gamma^{0}$ to a basic RA network $\Gamma=\left\{v_{i}\left(\rho_{i j}^{x} \otimes \rho_{i j}^{y}\right) v_{j}\right\}_{i, j=1}^{n}$ by setting $\rho_{i j}^{x}=\iota_{i j}^{x} \backslash\{\mathrm{m}, \mathrm{mi}\}$ and $\rho_{i j}^{y}=\iota_{i j}^{y} \backslash\{\mathrm{m}, \mathrm{mi}\}$. If $\Gamma$ is unsatisfiable, then neither is $\Delta$. Suppose $\Gamma$ is satisfiable and construct its canonical solution $\mathfrak{m}^{\Gamma}=\left(m_{i}^{\Gamma}\right)_{i=1}^{n}$ (cf. Figure 8).
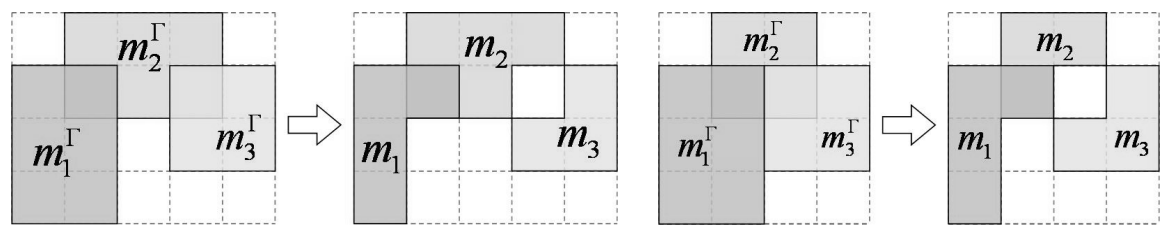

Figure 8: Illustration of Step 3: Deriving a solution $\mathfrak{m}$ of $\Delta$ from a canonical solution $\mathfrak{m}^{\Gamma}$ of $\Gamma$.

Step 3. This step tries to find a solution $\mathfrak{m}=\left(m_{i}\right)_{i=1}^{n}$ of the basic CDC network $\Delta$ such that $\mathcal{M}\left(m_{i}\right)=\mathcal{M}\left(m_{i}^{\Gamma}\right)$. Recall a basic CDC relation $\delta_{i j}$ is represented as a $3 \times 3$ Boolean matrix $\left(\left(\delta_{i j}\right)_{x y}\right)$. If $\mathfrak{m}$ is a solution, $m_{i}^{\circ} \cap\left(m_{j}\right)_{x y}=\varnothing$ holds for every $\left(\delta_{i j}\right)_{x y}=0$, where $\left(m_{j}\right)_{x y}$ is one of the nine tiles generated by $\mathcal{M}\left(m_{j}\right)$ (cf. Figure 3 ). This means, to make $\mathfrak{m}$ a solution to $\Delta$, we need to exclude all impossible cells from $m_{i}^{\Gamma}$. Set $T_{i}=\bigcup\left\{\left(m_{j}^{\Gamma}\right)_{x y}:\left(\delta_{i j}\right)_{x y}=0\right\}_{j=1}^{n}$. Let $m_{i}$ be the closure of $m_{i}^{\Gamma} \backslash T_{i}$ (cf. Figure 8 left).

Step 4. The last step checks whether $\mathfrak{m}=\left(m_{i}\right)_{i=1}^{n}$ is a solution of $\Delta$. If it is a solution, then $\mathfrak{m}$ must be a regular solution; if it is not, then we assert that $\Delta$ has no solution at all.

We note that other regular solutions may exist (cf. Figure 8 right). We can get all of them by repeating Steps 2 to 4 using every possible refinement of $\Gamma^{0}$.

\section{Appendix B. Proof of Theorem 22}

We only need to show the 'sufficiency' part. Similar to the cubic construction method for basic RCC8 constraints (cf. Li, 2006b and Section 2.2.1 of this paper), we construct a solution $\mathfrak{m}=\left(m_{i}\right)_{i=1}^{n}$ with an additional requirement that $\mathcal{M}\left(m_{i}\right)=m_{i}^{\Gamma}$ for each $1 \leq i \leq n$, where $\left\{m_{1}^{\Gamma}, \ldots, m_{n}^{\Gamma}\right\}$ is the canonical solution of $\Delta$. Recall that the coordinates of each corner point of a rectangle $m_{i}^{\Gamma}$ are integral. Assuming the ntpp-level $l(i)$ has been computed for each $1 \leq i \leq n$, we next describe the construction in detail. 


\section{Step 1. Selection of Control Points}

For each $v_{i}$ we select a set of control points $X_{i}$. First of all, each corner point in $C_{i}$ is a control point for $v_{i}$. We then select one (non-integral) point from each edge of $m_{i}^{\Gamma}$ and put the four points into $X_{i}$. Then, for any $j>i$ with $\theta_{i j}=\mathbf{E C}$ or PO select a point $P_{i j}$ from $m_{i}^{\Gamma} \cap m_{j}^{\Gamma}$ (which is nonempty because of the bipath-consistency of $\Theta \uplus \Gamma$ ), and put it into both $X_{i}$ and $X_{j}$. Note that $m_{i}^{\Gamma} \cap m_{j}^{\Gamma}$ could be a single point, or a line segment, or a rectangle. When choosing $P_{i j}$ from $m_{i}^{\Gamma} \cap m_{j}^{\Gamma}$, we require that each $P_{i j}$ is a fresh point that has not been chosen before and is not a corner point of any rectangle (unless $\Gamma_{i} \cap m_{j}^{\Gamma}$ is a singleton set). We write $\mathcal{P}$ for the set of all the control points.

\section{Step 2. Basic Regions Associated to Control Points}

For each control point $Q$, we construct a series of sectors $\left\{q^{i, k}: k=1, \cdots, 4\right\}_{i=1}^{n}$ and a series of squares $\left\{q^{(i)}\right\}_{i=1}^{n}$ (see Figure 9). We call these the basic regions associated to $Q$. Note that we use an upper case letter to denote a control point, and use the corresponding lower case letter (with indices) to denote basic regions. The sectors are chosen in this way as this allows us to distinguish up to four connecting regions in cases where $Q$ is a corner point (such as point $P$ in Figure3). The sectors completely fill all the disks.

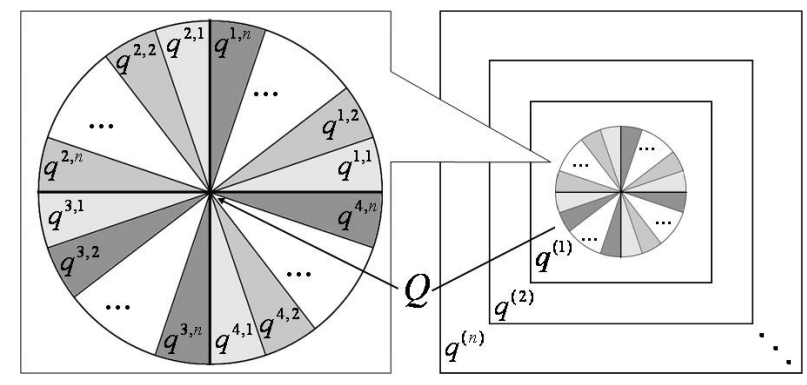

Figure 9: Basic regions of a control point $Q$.

For any two different control points, we require their outermost squares to be disjoint. Furthermore, a basic region must be small enough so that it is not crossed by the border of any $m_{i}^{\Gamma}$ of which $Q$ is not a boundary point.

\section{Step 3. Region Construction}

For each control point $Q$, set $q^{i}=\bigcup_{k=1}^{4} q^{i, k}$. Let

$$
\begin{aligned}
& a_{i}^{1}=m_{i}^{\Gamma} \cap \bigcup\left\{q^{i}: Q \in X_{i}\right\} \\
& a_{i}^{2}=a_{i}^{1} \cup\left(m_{i}^{\Gamma} \cap \bigcup\left\{q^{j}: \theta_{i j}=\mathbf{P O}, Q \in X_{i} \cap X_{j}\right\}\right) \\
& a_{i}^{3}=a_{i}^{2} \cup \bigcup\left\{a_{j}^{2}: \theta_{j i}=\mathbf{T P P} \text { or } \theta_{j i}=\mathbf{N T P P}\right\} \\
& a_{i}^{4}=a_{i}^{3} \cup \bigcup\left\{q^{(l(i))}: \theta_{j i}=\mathbf{N T P P}, Q \in a_{j}^{3}\right\}
\end{aligned}
$$

Set $m_{i}=a_{i}^{4}$ and $\mathfrak{m}=\left(m_{i}\right)_{i=1}^{n}$. It is easy to prove that $\mathfrak{m}$ satisfies all RCC8 constraints in $\Theta$. For example, suppose $\left(v_{i} \mathbf{D C} v_{j}\right)$ is a constraint in $\Theta$. Because (15) holds, we know $v_{i}$ and $v_{j}$ share no common conflict point, i.e. $C_{i} \cap C_{j}=\varnothing$. Due to the choice of control points for $v_{i}$ and $v_{j}$, we know 
$X_{i} \cap X_{j}$ is also empty. It is now easy to show that $m_{i} \cap m_{j}$ is empty and hence the DC constraint is satisfied.

To show $\mathfrak{m}$ also satisfies $\Gamma$, we need only prove $\mathcal{M}\left(m_{i}\right)=m_{i}^{\Gamma}$ for each $i$. It is clear that $a_{i}^{1}$ and $a_{i}^{2}$ are subsets of $m_{i}^{\Gamma}$. By the choice of $X_{i}$, we know $m_{i}^{\Gamma}=\mathcal{M}\left(a_{i}^{1}\right)=\mathcal{M}\left(a_{i}^{2}\right)$. If $\theta_{j i}=\mathbf{T P P}$ or NTPP, then $m_{j}^{\Gamma} \subseteq m_{i}^{\Gamma}$ by bipath-consistency. This implies $\mathcal{M}\left(a_{i}^{3}\right)=m_{i}^{\Gamma}$. Furthermore, if $\theta_{j i}=\mathbf{N T P P}$, we have $\left(m_{j}^{\Gamma}, m_{i}^{\Gamma}\right) \in \mathbf{d} \otimes \mathbf{d}$ by bipath-consistency. So for any control point $Q$ in $a_{j}^{3} \subseteq m_{j}^{\Gamma}, Q$ is also in the interior of $m_{i}^{\Gamma}$. Therefore, by the choice of basic regions, we know the outmost square $q^{(n)}$ at $Q$, hence $q^{(l(i))}$, is contained in $m_{i}^{\Gamma}$. Therefore, $\mathcal{M}\left(a_{i}^{4}\right)=m_{i}^{\Gamma}$. This proves that $\mathfrak{m}$ is a solution to $\Theta \uplus \Gamma$.

\section{Appendix C. The Reduction from 3SAT to $\mathbf{J S P}\left(\mathcal{B}_{r c c 8}, \mathcal{B}_{c d c}\right)$}

Let $\varphi=\bigwedge_{i=1}^{m} c_{j}$ be a 3SAT instance involving $n$ propositional variables $\left\{p_{k}\right\}_{k=1}^{n}$ and $m$ clauses. Assume that the $j$-th clause $c_{j}$ is $q_{i 1} \vee q_{i 2} \vee q_{i 3}$, where each $q_{i j}$ is a literal in $\left\{p_{k}\right\}_{k=1}^{n} \cup\left\{\neg p_{k}\right\}_{k=1}^{n}$. We construct a $\mathbf{J S P}\left(\mathcal{B}_{r c c 8}, \mathcal{B}_{c d c}\right)$ instance $\Theta_{\varphi} \uplus \Delta_{\varphi}$ and choose a particular regular solution $\mathfrak{m}$ of $\Delta_{\varphi}$ such that $\varphi$ is satisfiable iff $\mathfrak{m}$ is RA consistent with $\Theta_{\varphi}$.

There are three types of spatial variables in $\Theta_{\varphi} \uplus \Delta_{\varphi}$ : auxiliary variables (called grid variables) which are used to fix the relative locations of other variables, variables to simulate propositional variables, and variables to simulate propositional clauses.

\section{C.1 Grid Variables}

We introduce $10 \times n$ grid variables $G_{i j}(1 \leq i \leq 2 n, 1 \leq j \leq 5)$. The CDC constraints between these variables are specified as in Figure 10 (left). The RCC8 relation between two grid variables $G_{i j}, G_{i^{\prime} j^{\prime}}$ is $\mathbf{E C}$ if they are 4-neighbours, i.e. $\left\{\left|i-i^{\prime}\right|,\left|j-j^{\prime}\right|\right\}=\{0,1\}$. These $\mathbf{E C}$ constraints make sure that there is no gap between the MBRs of two neighbouring grid variables. This implies that there is at most one regular solution of $\Delta_{\varphi}$.

Grid variables are mainly used to locate other spatial variables. For a new variable $v$ and a grid variable $G_{i j}$, we say $v$ occupies $G_{i j}$ if $v \cap \mathcal{M}\left(G_{i j}\right)$ is nonempty, and its MBR is $\mathcal{M}\left(G_{i j}\right)$, i.e. $\mathcal{M}\left(v \cap \mathcal{M}\left(G_{i j}\right)\right)=\mathcal{M}\left(G_{i j}\right)$.

\begin{tabular}{|c|c|c|c|}
\hline$G_{1,1}$ & $G_{2,1}$ & $\cdots$ & $G_{5 n, 1}$ \\
\hline$G_{1,2}$ & $G_{2,2}$ & $\cdots$ & $G_{5 n, 2}$ \\
\hline$G_{1,3}$ & $G_{2,3}$ & $\cdots$ & $G_{5 n, 3}$ \\
\hline$G_{1,4}$ & $G_{2,4}$ & $\cdots$ & $G_{5_{n, 4}}$ \\
\hline$G_{1,5}$ & $G_{2,5}$ & $\cdots$ & $G_{5 n, 5}$ \\
\hline
\end{tabular}

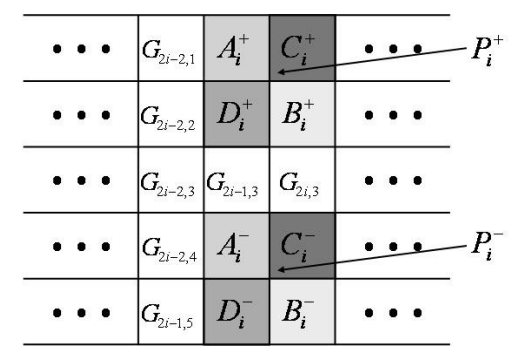

Figure 10: Grid and spatial variables for propositional variables.

\section{C.2 Spatial Variables for Propositional Variables}

For each propositional variable $p_{i}$ in $\varphi$, four spatial variables $A_{i}, B_{i}, C_{i}$ and $D_{i}$ are introduced such that each occupies two grid cells, but has an empty intersection with the interiors of the MBRs of 
all other grid variables. The corresponding grid cells are illustrated in Figure 10 (right). Take $A_{i}$ as example. By assigning the CDC constraints between $A_{i}$ and the grid variables, we require $A_{i}$ occupies $G_{2 i-1,1}$ and $G_{2 i-1,4}$, but is disjoint from the MBRs of all other grid variables. It is easy to see that $A_{i} \cap B_{i}$ contains at most two points, viz. $P_{i}^{+}$and $P_{i}^{-}$, and so does $C_{i} \cap D_{i}$.

As for the topological constraints, we require $A_{i} \mathbf{E C} B_{i}$ and $C_{i} \mathbf{E C} D_{i}$, and all other constraints are DC. The EC constraints imply that both $A_{i} \cap B_{i}$ and $C_{i} \cap D_{i}$ are nonempty. On the other hand, since $A_{i} \mathbf{D C} C_{i}$, we can conclude that $A_{i}$ and $B_{i}$ must share only one of $P_{i}^{+}$and $P_{i}^{-}$, while $C_{i}$ and $D_{i}$ share the other one.

\section{C.3 Spatial Variables for Propositional Clauses}

For each clause $\left(q_{j 1} \vee q_{j 2} \vee q_{j 3}\right)$ in $\varphi$, we introduce two new spatial variables $E_{j}$ and $F_{j}$, both occupy three grid cells. The precise occupied grid cells are set according to the variables and signs of $q_{j k}$. Figure 11 gives an example to illustrate the construction, where we assume $q_{j 1}=p_{i_{1}}, q_{j 2}=$ $\neg p_{i_{2}}, q_{j 3}=\neg p_{i_{3}}$. As for the topological constraints, we set the constraint between $E_{j}$ and $F_{j}$ as EC, and set all other constraints as DC. This implies $E_{j} \cap F_{j}$ contains at least one point of $P_{i_{1}}^{-}, P_{i_{2}}^{+}, P_{i_{3}}^{+}$. We claim that it is not the case that $A_{i_{1}} \cap B_{i_{1}}=\left\{P_{i_{1}}^{-}\right\}, A_{i_{2}} \cap B_{i_{2}}=\left\{P_{i_{2}}^{+}\right\}$and $A_{i_{3}} \cap B_{i_{3}}=\left\{P_{i_{3}}^{+}\right\}$. Otherwise, some DC constraint, e.g. that between $A_{i_{1}}$ and $E_{j}$, will be violated.

\section{C.4 The Regular Solution That May Be RA Consistent with $\Theta_{\varphi}$}

\begin{tabular}{l|l|l|l|l|l|l|l|l|l}
\hline$\cdots$ & $A_{i_{1}}^{+}$ & & $\cdots$ & $A_{i_{2}}^{+}$ & $E_{j}^{2}$ & $\cdots$ & $A_{i_{3}}^{+}$ & $E_{j}^{3}$ & $\cdots$ \\
\hline$\cdots$ & & $B_{i_{1}}^{+}$ & $\cdots$ & $F_{j}^{2}$ & $B_{i_{2}}^{+}$ & $\cdots$ & $F_{j}^{3}$ & $B_{i_{3}}^{+}$ & $\cdots$ \\
\hline$\cdots$ & & & $\cdots$ & & & $\cdots$ & & & $\cdots$ \\
\hline$\cdots$ & $A_{i_{1}}^{-}$ & $E_{j}^{1}$ & $\cdots$ & $A_{i_{2}}^{-}$ & & $\cdots$ & $A_{i_{3}}^{-}$ & & $\cdots$ \\
\hline$\cdots$ & $F_{j}^{1}$ & $B_{i_{1}}^{-}$ & $\cdots$ & & $B_{i_{2}}^{-}$ & $\cdots$ & & $B_{i_{3}}^{-}$ & $\cdots$ \\
\hline
\end{tabular}

Figure 11: Spatial variables for clauses.

We have now finished the construction. Note that $\Delta_{\varphi}$ is always satisfiable and there are exponentially many regular solutions of it (as there may be or may be not a gap between 4-neighbouring grid variables). However, the EC constraints between the 4-neighbouring grid variables imply that only the regular solution in which there is no gap between 4-neighbouring grid variables can be RA consistent with $\Theta_{\varphi}$. We denote this regular solution by $\mathfrak{m}$.

We next show that $\varphi$ is consistent iff $\Theta_{\varphi} \uplus \Delta_{\varphi}$ is consistent. Suppose $\Theta_{\varphi} \uplus \Delta_{\varphi}$ has a solution $\mathfrak{a}$. We define an assignment $\pi:\left\{p_{i}\right\}_{i=1}^{n} \rightarrow\{$ true, false $\}$ such that $\pi\left(p_{i}\right)=$ true iff $A_{i} \cap B_{i}=\left\{P_{i}^{+}\right\}$ in $\mathfrak{a}$. We can verify that $\pi$ satisfies $\varphi$. On the other hard, suppose $\pi$ is an assignment that satisfies $\varphi$. We prove that $\Theta_{\varphi} \uplus \Delta_{\varphi}$ has a solution. The idea is to introduce an instance of $\mathbf{J S P}\left(\mathcal{B}_{r c c 8}, \mathcal{B}_{r e c}\right)$, in which we have two spatial variables $A_{i}^{+}$and $A_{i}^{-}$instead of $A_{i}$ (also for $B_{i}, C_{i}, D_{i}$ ), and three variables $E_{j}^{k}(1 \leq k \leq 3)$ instead of $E_{j}$ (also for $F_{j}$ ). The RA constraints are set according to Figure 10 and Figure 11, while the RCC8 constraints are set by $\Theta_{\varphi}$ and $\pi$. It can be proven that this new joint network satisfies (15), and a solution can be obtained in cubic time. A solution of $\Theta_{\varphi} \uplus \Delta_{\varphi}$ can then be obtained by merging the related regions (e.g. merging $A_{i}^{+}$and $A_{i}^{-}$into $A_{i}$ ). 
The verification is straightforward. Therefore, $\varphi$ is satisfiable iff $\Theta_{\varphi} \uplus \Delta_{\varphi}$ is satisfiable, and thus $\varphi$ is satisfiable iff $\mathfrak{m}$ is RA consistent with $\Theta_{\varphi}$.

\section{Appendix D. The Reduction from Graph 3-Colouring to $\operatorname{JSP}\left(\mathcal{B}_{r c c 8}, \mathcal{B}_{c d c}\right)$}

Suppose $G=(V, E)$ is a graph. We construct an instance $\Theta_{G} \uplus \Delta_{G}$ of $\mathbf{J S P}\left(\mathcal{B}_{r c c 8}, \mathcal{B}_{c d c}\right)$ as follows. For each node $v_{i}$ in $V$, we construct a gadget with 10 spatial variables: $u_{i}^{k}(k=1,2, \cdots, 8), x_{i}$ and $y_{i}$. We first describe their CDC constraints. The basic CDC constraints between $u_{i}^{k}$ and $u_{i}^{k^{\prime}}$ are specified as in Figure 12 (i). For example, $\Delta_{G}$ contains the following basic CDC constraints

$$
u_{i}^{1}\left[\begin{array}{lll}
0 & 0 & 0 \\
1 & 0 & 0 \\
0 & 0 & 0
\end{array}\right] u_{i}^{2}, \quad u_{i}^{2}\left[\begin{array}{lll}
0 & 0 & 0 \\
0 & 0 & 1 \\
0 & 0 & 0
\end{array}\right] u_{i}^{1}, \quad u_{i}^{2}\left[\begin{array}{lll}
1 & 1 & 0 \\
1 & 1 & 0 \\
0 & 0 & 0
\end{array}\right] u_{i}^{7}, \quad u_{i}^{7}\left[\begin{array}{lll}
0 & 0 & 0 \\
0 & 1 & 1 \\
0 & 1 & 1
\end{array}\right] u_{i}^{2} .
$$

Note that, the induced RA constraint between $u_{i}^{2 l+1}$ and $u_{i}^{2 l+2}$ for $l=0,1,2$ is $(\mathrm{b} \cup \mathrm{m}) \otimes$ eq. The basic CDC constraints between $x_{i}$ and $y_{i}$ are specified as in Figure 12 (ii), i.e.

$$
x_{i}\left[\begin{array}{lll}
0 & 0 & 0 \\
1 & 1 & 0 \\
0 & 0 & 0
\end{array}\right] y_{i}, \quad y_{i}\left[\begin{array}{lll}
0 & 0 & 0 \\
0 & 1 & 1 \\
0 & 0 & 0
\end{array}\right] x_{i} .
$$

The CDC constraints concerning $x_{i}, y_{i}$ and $u_{i}^{k}$ are specified as follows.

$$
\begin{aligned}
& x_{i}\left[\begin{array}{lll}
0 & 0 & 0 \\
0 & 1 & 0 \\
0 & 0 & 1
\end{array}\right] u_{i}^{1}, \quad x_{i}\left[\begin{array}{lll}
0 & 0 & 0 \\
1 & 0 & 0 \\
0 & 0 & 1
\end{array}\right] u_{i}^{2}, \quad x_{i}\left[\begin{array}{lll}
1 & 0 & 0 \\
0 & 1 & 0 \\
0 & 0 & 1
\end{array}\right] u_{i}^{3}, \quad x_{i}\left[\begin{array}{ccc}
1 & 0 & 0 \\
1 & 0 & 0 \\
0 & 0 & 1
\end{array}\right] u_{i}^{4}, \\
& x_{i}\left[\begin{array}{lll}
1 & 0 & 0 \\
0 & 1 & 0 \\
0 & 0 & 0
\end{array}\right] u_{i}^{5}, \quad x_{i}\left[\begin{array}{lll}
1 & 0 & 0 \\
1 & 0 & 0 \\
0 & 0 & 0
\end{array}\right] u_{i}^{6}, \quad x_{i}\left[\begin{array}{lll}
1 & 0 & 0 \\
0 & 0 & 0 \\
0 & 0 & 1
\end{array}\right] u_{i}^{k} \quad(k=7,8), \\
& u_{i}^{k}\left[\begin{array}{ccc}
0 & 0 & 0 \\
0 & 1 & 0 \\
0 & 0 & 0
\end{array}\right] x_{i} \quad(k \neq 6), \quad u_{i}^{6}\left[\begin{array}{ccc}
0 & 0 & 0 \\
0 & 0 & 1 \\
0 & 0 & 0
\end{array}\right] x_{i} \\
& y_{i}\left[\begin{array}{lll}
0 & 0 & 0 \\
0 & 0 & 1 \\
0 & 0 & 1
\end{array}\right] u_{i}^{1}, \quad y_{i}\left[\begin{array}{lll}
0 & 0 & 0 \\
0 & 1 & 0 \\
0 & 0 & 1
\end{array}\right] u_{i}^{2}, \quad y_{i}\left[\begin{array}{lll}
1 & 0 & 0 \\
0 & 0 & 1 \\
0 & 0 & 1
\end{array}\right] u_{i}^{3}, \quad y_{i}\left[\begin{array}{lll}
1 & 0 & 0 \\
0 & 1 & 0 \\
0 & 0 & 1
\end{array}\right] u_{i}^{4}, \\
& y_{i}\left[\begin{array}{lll}
1 & 0 & 0 \\
0 & 0 & 1 \\
0 & 0 & 0
\end{array}\right] u_{i}^{5}, \quad y_{i}\left[\begin{array}{lll}
1 & 0 & 0 \\
0 & 1 & 0 \\
0 & 0 & 0
\end{array}\right] u_{i}^{6}, \quad y_{i}\left[\begin{array}{lll}
1 & 0 & 0 \\
0 & 0 & 0 \\
0 & 0 & 1
\end{array}\right] u_{i}^{k} \quad(k=7,8), \\
& u_{i}^{k}\left[\begin{array}{ccc}
0 & 0 & 0 \\
0 & 1 & 0 \\
0 & 0 & 0
\end{array}\right] y_{i} \quad(2 \leq k \leq 8), \quad u_{i}^{1}\left[\begin{array}{lll}
0 & 0 & 0 \\
1 & 0 & 0 \\
0 & 0 & 0
\end{array}\right] y_{i} .
\end{aligned}
$$

Figure 12 (ii) illustrates a regular solution of $u_{i}^{k}, x_{i}$ and $y_{i}$, where $u_{i}^{1}$ meets $u_{i}^{2}$ while there is a gap between $u_{i}^{3}$ and $u_{i}^{4}$, and between $u_{i}^{5}$ and $u_{i}^{6}$. We note that there are in total eight different regular solutions when the network is restricted to the gadget of $v_{i}$. 

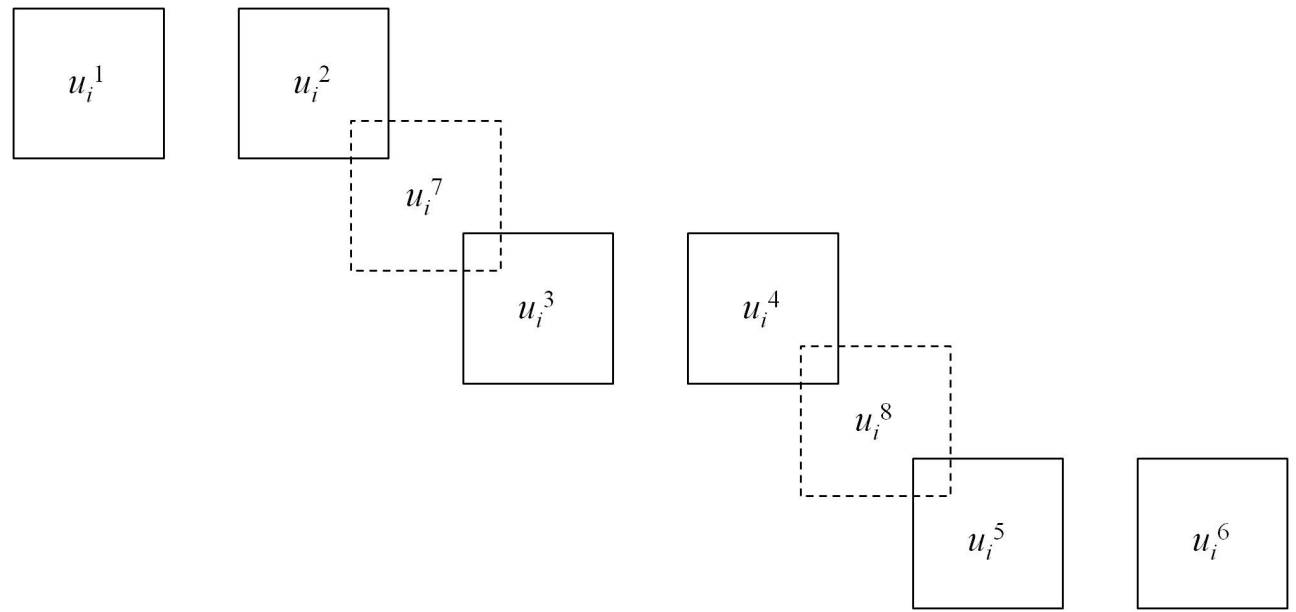

(i)
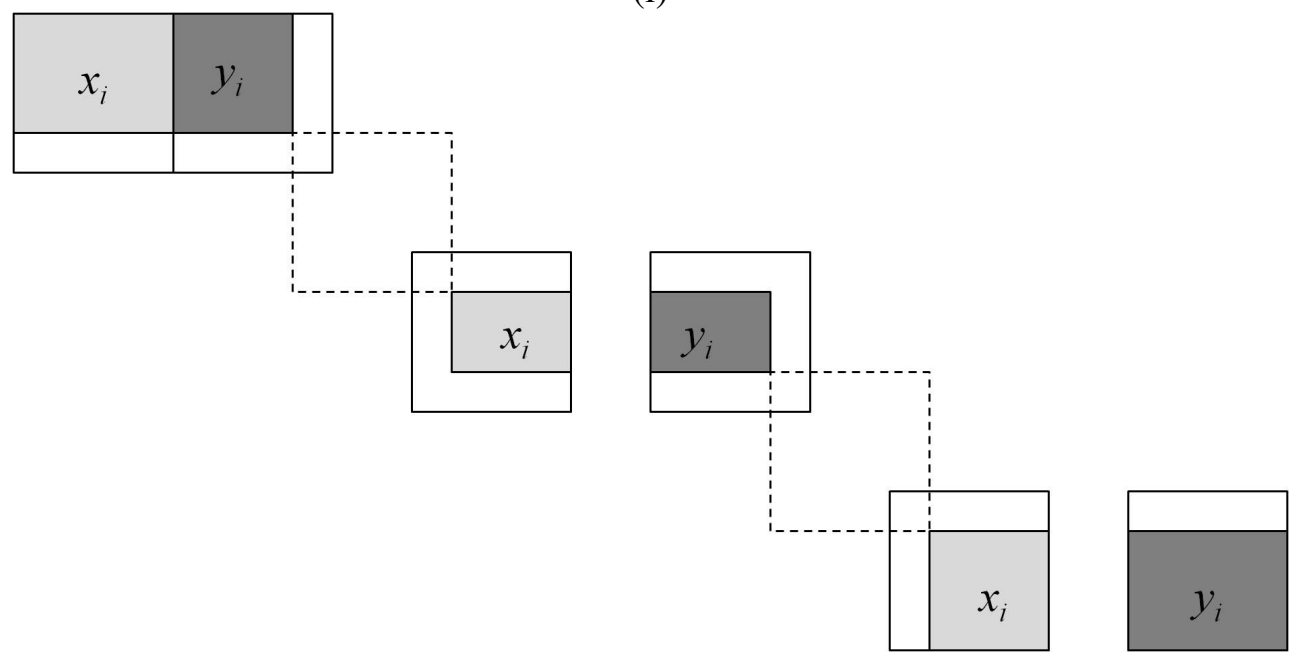

(ii)

Figure 12: Illustrations of the CDC constraints: (i) constraints between $u_{i}^{k}(k=1,2, \cdots, 8)$; (ii) constraints relating $x_{i}, y_{i}$. Note we use dashed squares to denote variables $u_{i}^{7}$ and $u_{i}^{8}$, which 'connect' two other variables. Note that both $x_{i}$ and $y_{i}$ have three disjoint parts. 
The RCC 8 constraint between any two variables is either $\mathbf{E C}$ or DC. We require $x_{i} \mathbf{E C} y_{i}$. This is realisable only if $u_{i}^{2 l+1}$ meets $u_{i}^{2 l+2}$ in $x$-direction for at least one $l \in\{0,1,2\}{ }^{4}$ We use this fact to mimic that the node $v_{i} \in V$ is coloured with one of the three colours. The RCC8 constraints of the remaining pairs of variables are all specified as DC. ${ }^{5}$

The gadgets for all nodes in $V$ are horizontally aligned, as illustrated in Figure 13.
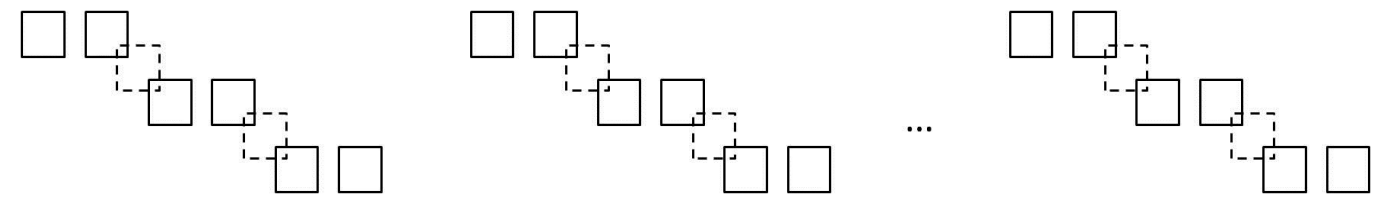

Figure 13: Illustrations of the gadgets for all nodes in $V$

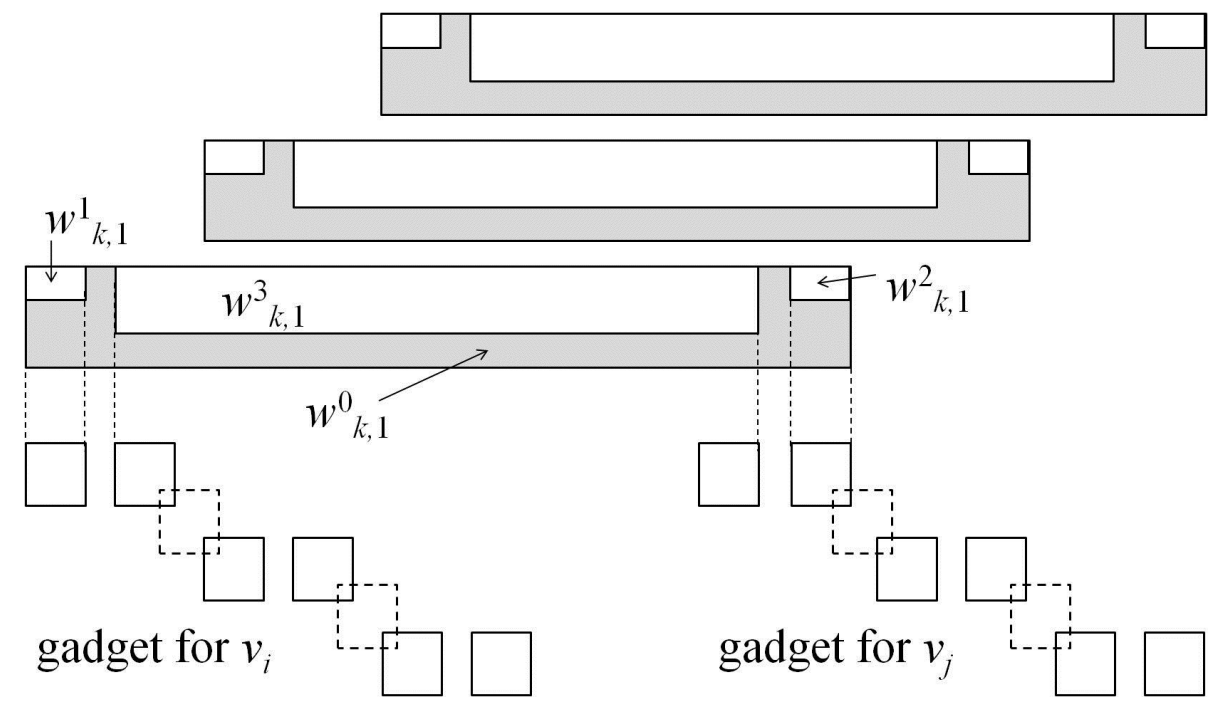

Figure 14: Illustrations of the gadget for edge $e_{k}=\left(v_{i}, v_{j}\right)$, where dashed lines suggest that corresponding edges are aligned according to proper CDC constraints.

We then devise the gadgets for edges in graph $G$. Let $e_{k}=\left(v_{i}, v_{j}\right)$ be an edge in $E$. For each colour $l \in\{0,1,2\}$, we introduce four variables $w_{k, l}^{0}, w_{k, l}^{1}, w_{k, l}^{2}$ and $w_{k, l}^{3}$ as well as constraints to guarantee that $u_{i}^{2 l+1}$ cannot meet $u_{i}^{2 l+2}$ if $u_{j}^{2 l+1}$ meets $u_{j}^{2 l+2}$, which corresponds to that nodes $v_{i}$ and $v_{j}$ cannot both have colour $l$ (because $e_{k}$ is an edge in $G$ ). The CDC constraints are specified as in Figure 14. We note that $w_{k, l}^{1}$ meets $w_{k, l}^{3}$ iff $u_{i}^{2 l+1}$ meets $u_{i}^{2 l+2}$, and $w_{k, l}^{3}$ meets $w_{k, l}^{2}$ iff $u_{j}^{2 l+1}$ meets $u_{j}^{2 l+2}$, all in $x$-direction. By the CDC constraints we can show that $\mathcal{M}\left(w_{k, l}^{3}\right)$ is contained in $\mathcal{M}\left(w_{k, l}^{0}\right)$. This implies that there is either a gap between $w_{k, l}^{1}$ and $w_{k, l}^{3}$ or a gap between $w_{k, l}^{3}$ and $w_{k, l}^{2}$. In other words, $w_{k, l}^{1}$ meets $w_{k, l}^{3}$ and $w_{k, l}^{3}$ meets $w_{k, l}^{2}$ cannot happen simultaneously. By the

4. If there are more than one $l$ such that $u_{i}^{2 l+1}$ meets $u_{i}^{2 l+2}$ in $x$-direction, we always choose the smallest such $l$ as the 'colour' of the node $v_{i}$.

5. Note that $u_{i}^{1} \mathbf{D C} u_{i}^{2}$ together with the CDC relations between $u_{i}^{1}$ and $u_{i}^{2}$ does not necessarily imply that $u_{i}^{1}$ should precede $u_{i}^{2}$ in $x$-direction. That is, $u_{i}^{1}$ could still meet $u_{i}^{2}$ in $x$-direction in this case. 
constraints enforcing the dashed lines we know $u_{i}^{2 l+1}$ cannot meet $u_{i}^{2 l+2}$ if $u_{j}^{2 l+1}$ meets $u_{j}^{2 l+2}$, and vice versa.

Note that we need to complete both $\Theta$ and $\Delta$. While unspecified CDC constraints can be easily deduced from these figures, unspecified RCC8 constraints are all DC.

It is not hard to verify that graph $G$ is 3-colourable iff the joint network $\Theta_{G} \uplus \Delta_{G}$ is consistent. The idea is that, if $\pi: V \rightarrow\{0,1,2\}$ is a 3-colouring of $G$, then we may construct a solution of $\Theta_{G} \uplus \Delta_{G}$ in which the RA relation between $u_{i}^{2 l+1}$ and $u_{i}^{2 l+2}$ is $\mathrm{m} \otimes$ eq if $\pi(i)=l$, and is $\mathrm{b} \otimes$ eq otherwise. This guarantees that $x_{i}$ and $y_{i}$ are realisable. The fact that no incident nodes in $G$ have the same colour implies that all $w_{k, l}^{r}$ are realisable. On the other hand, if $\Theta_{G} \uplus \Delta_{G}$ is satisfiable, then at least one pair in $\left\{\left(u_{i}^{1}, u_{i}^{2}\right),\left(u_{i}^{3}, u_{i}^{4}\right),\left(u_{i}^{5}, u_{i}^{6}\right)\right\}$ should have RA relation $\mathrm{m} \otimes$ eq (otherwise, $x_{i} \mathbf{E C} y_{i}$ is violated). Define $\pi: V \rightarrow\{0,1,2\}$ by $\pi\left(v_{i}\right)=\min \left\{l: u_{i}^{2 l+1} \mathrm{~m} \otimes\right.$ eq $\left.u_{i}^{2 l+2}\right\}$. It can be verified that $\pi$ is a 3-colouring of $G$ due to the fact that $w_{k, l}^{r}$ are realisable.

Now we have completed the reduction from Graph 3-Colouring to $\mathbf{J S P}\left(\mathcal{B}_{r c c 8}, \mathcal{B}_{c d c}\right)$. We note that the $\mathbf{E C}$ constraints can also be interpreted in terms of RCC ${ }^{\prime}$. This means that $\Theta_{G} \uplus \Delta_{G}$ can also be regarded as an instance of $\mathbf{J S P}\left(\mathcal{B}_{r c c 8}, \mathcal{B}_{c d c}\right)$. Therefore, we have also provided a reduction from Graph 3-Colouring to $\mathbf{J S P}\left(\mathcal{B}_{r c c 8^{\prime}}, \mathcal{B}_{c d c}\right)$.

\section{References}

Allen, J. F. (1983). Maintaining knowledge about temporal intervals. Communications of the ACM, 26(11), 832-843.

Balbiani, P., Condotta, J.-F., \& Fariñas del Cerro, L. (1999). A new tractable subclass of the rectangle algebra. In Dean, D. (Ed.), Proceedings of the Sixteenth International Joint Conference on Artificial Intelligence (IJCAI-99), pp. 442-447. Morgan Kaufmann.

Bodirsky, M., \& Kára, J. (2010). The complexity of temporal constraint satisfaction problems. J. ACM, 57(2).

Borgo, S., Guarino, N., \& Masolo, C. (1996). A pointless theory of space based on strong connection and congruence. In Aiello, L. C., Doyle, J., \& Shapiro, S. C. (Eds.), KR, pp. 220-229. Morgan Kaufmann.

Chen, J., Cohn, A. G., Liu, D., Wang, S., Ouyang, J., \& Yu, Q. (2013). A survey of qualitative spatial representations. The Knowledge Engineering Review, FirstView, 1-31.

Cicerone, S., \& Di Felice, P. (2004). Cardinal directions between spatial objects: the pairwiseconsistency problem. Information Sciences, 164(1-4), 165-188.

Cohn, A. G., \& Renz, J. (2008). Qualitative spatial reasoning. In van Harmelen, F., Lifschitz, V., \& Porter, B. (Eds.), Handbook of Knowledge Representation. Elsevier.

Cohn, A. G., Renz, J., \& Sridhar, M. (2012). Thinking inside the box: A comprehensive spatial representation for video analysis. In $K R$, pp. 588-592.

Cohn, A. G., \& Varzi, A. C. (1999). Modes of connection. In Freksa, C., \& Mark, D. M. (Eds.), COSIT, Vol. 1661 of Lecture Notes in Computer Science, pp. 299-314. Springer.

Davis, E. (2013). Qualitative spatial reasoning in interpreting text and narrative. Spatial Cognition \& Computation, 13(4), 264-294. 
Davis, E., Gotts, N. M., \& Cohn, A. G. (1999). Constraint networks of topological relations and convexity. Constraints, 4(3), 241-280.

Düntsch, I., Wang, H., \& McCloskey, S. (2001). A relation-algebraic approach to the Region Connection Calculus. Theoretical Computer Science, 255, 63-83.

Egenhofer, M. J., \& Mark, D. M. (1995). Naive geography. In Frank, A., \& Kuhn, W. (Eds.), COSIT-95, pp. 1-15. Springer.

Falomir, Z. (2012). Qualitative distances and qualitative description of images for indoor scene description and recognition in robotics. AI Communications, 25(4), 387-389.

Freksa, C. (1992). Temporal reasoning based on semi-intervals. Artificial Intelligence, 54(1), 199227.

Gabelaia, D., Kontchakov, R., Kurucz, Á., Wolter, F., \& Zakharyaschev, M. (2005). Combining spatial and temporal logics: Expressiveness vs. complexity. Journal of Artificial Intelligence Research, 23, 167-243.

Ge, X., \& Renz, J. (2013). Representation and reasoning about general solid rectangles. In Rossi, F. (Ed.), IJCAI. IJCAI/AAAI.

Gerevini, A., \& Renz, J. (2002). Combining topological and size information for spatial reasoning. Artificial Intelligence, 137(1), 1-42.

Gerevini, A., \& Nebel, B. (2002). Qualitative spatio-temporal reasoning with rcc-8 and allen's interval calculus: Computational complexity. In ECAI, pp. 312-316.

Goyal, R., \& Egenhofer, M. (1997). The direction-relation matrix: A representation for directions relations between extended spatial objects. In The Annual Assembly and the Summer Retreat of University Consortium for Geographic Information Systems Science.

Goyal, R., \& Egenhofer, M. (2001). Similarity of cardinal directions. In Jensen, C., Schneider, M., Seeger, B., \& Tsotras, V. (Eds.), Proceedings of the 7th International Symposium on Advances in Spatial and Temporal Databases (SSTD-01), pp. 36-58. Springer.

Hirsch, R. (1999). A finite relation algebra with undecidable network satisfaction problem. Logic Journal of the IGPL, 7(4), 547-554.

Jonsson, P., \& Krokhin, A. A. (2004). Complexity classification in qualitative temporal constraint reasoning. Artificial Intelligence, 160(1-2), 35-51.

Kontchakov, R., Nenov, Y., Pratt-Hartmann, I., \& Zakharyaschev, M. (2011). On the decidability of connectedness constraints in 2D and 3D Euclidean spaces. In IJCAI, pp. 957-962.

Li, S. (2006a). Combining topological and directional information: First results. In Lang, J., Lin, F., \& Wang, J. (Eds.), Proceedings of the First International Conference on Knowledge Science, Engineering and Management (KSEM-06), pp. 252-264. Springer.

Li, S. (2006b). On topological consistency and realization. Constraints, 11(1), 31-51.

Li, S. (2007). Combining topological and directional information for spatial reasoning. In Veloso, M. (Ed.), Proceedings of the 20th International Joint Conference on Artificial Intelligence (IJCAI-07), pp. 435-440. AAAI.

Li, S., \& Cohn, A. G. (2012). Reasoning with topological and directional spatial information. Computational Intelligence, 28(4), 579-616. 
Li, S., \& Liu, W. (2014). Cardinal directions: A comparison of direction relation matrix and objects interaction matrix. International Journal of Geographical Information Science, accepted for publication, doi: http://dx.doi.org/10.1080/13658816.2014.954580.

Li, S., Liu, W., \& Wang, S. (2013). Qualitative constraint satisfaction problems: An extended framework with landmarks. Artificial Intelligence, 201, 32-58.

Ligozat, G. (1994). Tractable relations in temporal reasoning: pre-convex relations. In ECAI-94. Workshop on Spatial and Temporal Reasoning, pp. 99-108.

Ligozat, G., \& Renz, J. (2004). What is a qualitative calculus? A general framework. In Zhang, C., Guesgen, H., \& Yeap, W.-K. (Eds.), PRICAI-04, pp. 53-64. Springer.

Liu, W., \& Li, S. (2011). Reasoning about cardinal directions between extended objects: The NPhardness result. Artificial Intelligence, 175(18), 2155-2169.

Liu, W., Li, S., \& Renz, J. (2009). Combining RCC-8 with qualitative direction calculi: Algorithms and complexity. In Boutilier, C. (Ed.), Proceedings of the Twenty-first International Joint Conference on Artificial Intelligence (IJCAI-09), pp. 854-859.

Liu, W., Zhang, X., Li, S., \& Ying, M. (2010). Reasoning about cardinal directions between extended objects. Artificial Intelligence, 174(12-13), 951-983.

Nebel, B. (1995). Computational properties of qualitative spatial reasoning: First results. In KI-95, pp. 233-244, Berlin, Germany. Springer-Verlag.

Nebel, B., \& Bürckert, H.-J. (1995). Reasoning about temporal relations: A maximal tractable subclass of Allen's interval algebra. Journal of the ACM, 42(1), 43-66.

Randell, D. A., Cui, Z., \& Cohn, A. G. (1992). A spatial logic based on regions and connection. In KR-92, pp. 165-176.

Renz, J. (1999). Maximal tractable fragments of the Region Connection Calculus: A complete analysis. In Dean, D. (Ed.), Proceedings of the Sixteenth International Joint Conference on Artificial Intelligence (IJCAI-99), pp. 448-454. Morgan Kaufmann.

Renz, J. (2002). Qualitative spatial reasoning with topological information, Vol. 2293 of Lecture Notes in Artificial Intelligence. Springer-Verlag, Berlin, Germany.

Renz, J., \& Nebel, B. (1999). On the complexity of qualitative spatial reasoning: A maximal tractable fragment of the Region Connection Calculus. Artificial Intelligence, 108, 69-123.

Schneider, M., Chen, T., Viswanathan, G., \& Yuan, W. (2012). Cardinal directions between complex regions. ACM Transactions on Database Systems, 37(2), 8:1-8:40.

Schockaert, S., \& Li, S. (2012). Convex solutions of RCC8 networks. In ECAI, pp. 726-731.

Schockaert, S., \& Li, S. (2013). Combining RCC5 relations with betweenness information. In IJCAI, pp. 1083-1089.

Shi, H., Jian, C., \& Krieg-Brückner, B. (2010). Qualitative spatial modelling of human route instructions to mobile robots. In ACHI, pp. 1-6.

Sistla, A., \& Yu, C. (2000). Reasoning about qualitative spatial relationships. Journal of Automated Reasoning, 25(4), 291-328.

Skiadopoulos, S., \& Koubarakis, M. (2005). On the consistency of cardinal direction constraints. Artificial Intelligence, 163(1), 91-135. 
Sridhar, M., Cohn, A. G., \& Hogg, D. C. (2011). From video to RCC8: Exploiting a distance based semantics to stabilise the interpretation of mereotopological relations. In COSIT, pp. $110-125$.

Vilain, M. B., \& Kautz, H. A. (1986). Constraint propagation algorithms for temporal reasoning. In AAAI, pp. 377-382.

Whitehead, A. (1929). Process and Reality: An Essay in Cosmology. Cambridge University Press, Cambridge.

Wölfl, S., \& Westphal, M. (2009). On combinations of binary qualitative constraint calculi. In Boutilier, C. (Ed.), Proceedings of the Twenty-first International Joint Conference on Artificial Intelligence (IJCAI-09), pp. 967-972.

Wolter, D., \& Wallgrün, J. (2012). Qualitative spatial reasoning for applications: New challenges and the sparq toolbox. In Hazarika, M. (Ed.), Qualitative Spatio-Temporal Representation and Reasoning: Trends and Future Directions, pp. 336-362.

Wolter, D., Dylla, F., Wölfl, S., Wallgrün, J. O., Frommberger, L., Nebel, B., \& Freksa, C. (2008). Sailaway: Spatial cognition in sea navigation. KI, 22(1), 28-30.

Wolter, F., \& Zakharyaschev, M. (2000). Spatial reasoning in RCC-8 with boolean region terms. In ECAI, pp. 244-250.

Zhang, P., \& Renz, J. (2014). Qualitative spatial representation and reasoning in angry birds: the extended rectangle algebra. In $K R$.

Zhang, X., Liu, W., Li, S., \& Ying, M. (2008). Reasoning with cardinal directions: An efficient algorithm. In Fox, D., \& Gomes, C. (Eds.), Proceedings of the Twenty-Third AAAI Conference on Artificial Intelligence (AAAI-08), pp. 435-440. AAAI. 